\title{
The second Painlevé equation, a related nonautonomous semidiscrete equation, and a limit to the first Painlevé equation: scalar and matrix cases
}

\author{
Andrew Pickering ${ }^{1}$, Pilar R Gordoa ${ }^{1}$, Jonathan AD Wattis ${ }^{2}$, \\ ${ }^{1}$ Área de Matemática Aplicada, ESCET, Universidad Rey Juan Carlos, \\ C/Tulipán s/n, 28933 Móstoles, Madrid, Spain \\ andrew.pickering@urjc.es pilar.gordoa@urjc.es \\ ${ }^{2}$ School of Mathematical Sciences, University of Nottingham, \\ University Park, Nottingham NG7 2RD, UK \\ Jonathan.Wattis@nottingham.ac.uk
}

\begin{abstract}
In this paper we consider the matrix nonautonomous semidiscrete (or lattice) equation $\frac{\mathrm{d}}{\mathrm{d} t} \mathbf{U}_{n}=(2 n-1)\left(\mathbf{U}_{n+1}-\mathbf{U}_{n-1}\right)^{-1}$, as well as the scalar case thereof. This equation was recently derived in the context of auto-Bäcklund transformations for a matrix partial differential equation. We use asymptotic techniques to reveal a connection between this equation and the matrix (or, as appropriate, scalar) first Painlevé equation. In the matrix case, we also discuss our asymptotic analysis more generally, as well as considering a component-wise approach. In addition, Hamiltonian formulations of the matrix first and second Painlevé equations are given, as well as a discussion of classes of solutions of the matrix second Painlevé equation.
\end{abstract}

Keywords: matrix semidiscrete equations, asymptotic behaviour, Hamiltonian formulations of matrix Painlevé equations, solutions of matrix second Painlevé equation, integrable systems.

\section{Highlights:}

- we derive Hamiltonian formulations for matrix $P_{I}$ \& matrix $P_{I I}$ systems of equations

- asymptotic reductions are used to derive $P_{I}$ from a semidiscrete equation

- derivation generalised to matrix form using general and component-wise expansions

- solutions of matrix $P_{I I}$ are elucidated using combinations of autoBacklund Transforms 


\section{Introduction}

There has been, over the last twenty years or so, a surge in interest in Painlevé hierarchies and their properties. In [1], Gordoa, Pickering and Zhu introduced the first examples of matrix Painlevé hierarchies, these being matrix versions of the first and second Painlevé $\left(P_{I}\right.$ and $\left.P_{I I}\right)$ hierarchies. Amongst other results presented in [1], they gave auto-Bäcklund transformations (aBTs) for their matrix $P_{I I}$ hierarchy and, for the particular case of the matrix $P_{I I}$ equation as presented in [1], used these auto-Bäcklund transformations to derive a discrete matrix $P_{I}$ equation; these results for the matrix $P_{I I}$ equation generalized their previous reults given in $[2,3]$.

The matrix $P_{I I}$ equation presented in [1] has the form

$$
\mathbf{u}_{x x}-2 \mathbf{u}^{3}+c_{0} \mathbf{u}+\mathbf{u} \mathbf{E}_{0}+\mathbf{E}_{0} \mathbf{u}+2 g_{0} x \mathbf{u}-\alpha \mathbf{I}=\mathbf{0},
$$

where $\mathbf{u}=\left(u_{i j}\right)$ is an $m \times m$ matrix of functions $u_{i j}(x), \mathbf{E}_{0}=\left(e_{i j}\right)$ is an arbitrary constant $m \times m$ matrix, and $c_{0}, g_{0}$ and $\alpha$ are arbitrary scalar constants. As noted in [1], the term $c_{0} \mathbf{u}$ can be absorbed in the terms $\mathbf{u E}_{0}+\mathbf{E}_{0} \mathbf{u}$, but we prefer not to do so: this then means that in the reduction to the scalar case we take $\mathbf{E}_{0}=0$ rather than $\mathbf{E}_{0}$ to be a multiple of the identity matrix. In the case $g_{0} \neq 0$ we may assume without loss of generality (using a change of independent and dependent variables) that $g_{0}=-1 / 2$, and so may take our equation in the form

$$
\mathbf{u}_{x x}-2 \mathbf{u}^{3}+c_{0} \mathbf{u}+\mathbf{u} \mathbf{E}_{0}+\mathbf{E}_{0} \mathbf{u}-x \mathbf{u}-\alpha \mathbf{I}=\mathbf{0} .
$$

In the special case where $\mathbf{E}_{0}=0$ or is a multiple of the identity matrix, this last equation is equivalent (using a shift on $x$ ) to the matrix $P_{I I}$ equation given in $[4,5]$, that is

$$
\mathbf{u}_{x x}=2 \mathbf{u}^{3}+x \mathbf{u}+\alpha \mathbf{I} .
$$

In a recent paper [6], Gordoa and Pickering presented a matrix partial differential equation (PDE) whose structure mirrors that of the matrix $P_{I I}$ equation and to which it has a simple reduction, and which admits aBTs of a form similar to those of matrix $P_{I I}$. They then used these aBTs to derive the nonautonomous semidiscrete matrix equation

$$
\frac{\mathrm{d}}{\mathrm{d} t} \mathbf{U}_{n}=(2 n-1)\left(\mathbf{U}_{n+1}-\mathbf{U}_{n-1}\right)^{-1} .
$$

That aBTs for ordinary differential equations (ODEs) may be used to derive discrete equations was shown in [7, 8]. It is worth noting, however, that in contrast to [7, 8], in [6] these ideas were used within the context of aBTs for PDEs, and moreover the result was a semidiscrete equation as opposed to a purely discrete equation. It is to this last equation, i.e., (1.4), as well as the scalar version thereof, that the greater part of this paper is devoted.

Equation (1.4) can also be derived from the aBTs of the matrix $P_{I I}$ equation with $g_{0} \neq 0$ (it is this case which is the matrix analogue of the second Painlevé equation). It is perhaps this more familiar context, rather than its derivation from PDE aBTs, which explains the significance of (1.4). The technique published in [7, 8], whereby aBTs of integrable ODEs are used to derive new integrable discrete equations, has led to the discovery of a number of new 
discrete equations which have proved to be of considerable interest. The interest in the lattice equation (1.4) is due to its novel derivation within the context of Painlevé equations and their aBTs. This context immediately brings to mind questions such as that of its continuum limits, a question studied for similarly-derived purely discrete equations.

Semidiscrete equations have of course been well-studied in the literature, with the integrability of such equations being a topic that stretches back to the beginnings of soliton theory, e.g., with the discovery of the Toda lattice. Many different aspects of integrable lattice equations have been explored over the last half-century, far too many to mention here. These include for instance inverse scattering, Hirota bilinear form, continuum limits and exact solutions. Also for example, non-isospectral terms in lattice equations, as considered for instance in $[9,10,11,12]$, have proved to be of particular interest due to the relationship with discrete Painlevé equations. In addition, we note the presentation of a procedure to find solutions of lattice equations via the Casoratian technique in [13], where the derivation of rational solutions - as noted therein, a class of solutions that Painlevé equations may also have - as well as of mixed rational-soliton solutions was considered. In the study of semidiscrete equations it is of course natural to seek similar results to those known for continuous systems. Thus we consider the possibility of general results for lump solutions, in continuous systems, as discussed in [14] (see also [15]), and in semidiscrete equations (see, e.g., [16]).

The derivation of (1.4) from the aBTs of matrix $P_{I I}$ is explained in [6], though not completely explicitly. This derivation is worth considering, as it is somewhat curious, and so - given also that (1.4) and the scalar version thereof together form the main topic of this paper - we briefly describe it here. We begin by writing matrix $P_{I I}$ with $g_{0}=-1 / 2$, i.e., equation (1.2), as the system

$$
\mathbf{u}_{x x}-\mathbf{u y}_{x}-\mathbf{y}_{x} \mathbf{u}+c_{0} \mathbf{u}+\mathbf{u} \mathbf{E}_{0}+\mathbf{E}_{0} \mathbf{u}-x \mathbf{u}-\alpha \mathbf{I}=\mathbf{0}, \quad \mathbf{y}_{x}=\mathbf{u}^{2},
$$

this being the corresponding ODE reduction of the matrix PDE considered in [6]. We then observe that this system has the aBTs

$$
\begin{aligned}
& \mathbf{u}=-\mathbf{v}+\frac{1}{2}(\alpha+\widetilde{\alpha})\left(\mathbf{v}_{x}-\mathbf{z}_{x}+\frac{1}{2} c_{0} \mathbf{I}+\mathbf{E}_{0}-\frac{1}{2} \mathbf{I} x\right)^{-1}, \\
& \mathbf{y}=\mathbf{z}-\frac{1}{2}(\alpha+\widetilde{\alpha})\left(\mathbf{v}_{x}-\mathbf{z}_{x}+\frac{1}{2} c_{0} \mathbf{I}+\mathbf{E}_{0}-\frac{1}{2} \mathbf{I} x\right)^{-1}, \\
& \alpha=\widetilde{\alpha}-1,
\end{aligned}
$$

and

$$
\begin{aligned}
& \widehat{\mathbf{u}}=-\mathbf{v}+\frac{1}{2}(\widehat{\alpha}+\widetilde{\alpha})\left(-\mathbf{v}_{x}-\mathbf{z}_{x}+\frac{1}{2} c_{0} \mathbf{I}+\mathbf{E}_{0}-\frac{1}{2} \mathbf{I} x\right)^{-1}, \\
& \widehat{\mathbf{y}}=\mathbf{z}+\frac{1}{2}(\widehat{\alpha}+\widetilde{\alpha})\left(-\mathbf{v}_{x}-\mathbf{z}_{x}+\frac{1}{2} c_{0} \mathbf{I}+\mathbf{E}_{0}-\frac{1}{2} \mathbf{I} x\right)^{-1}, \\
& \widehat{\alpha}=\widetilde{\alpha}+1,
\end{aligned}
$$

which map from solutions $(\mathbf{v}, \mathbf{z}, \widetilde{\alpha})$ to solutions $(\mathbf{u}, \mathbf{y}, \alpha)$ of $(1.5)$, and from solutions $(\mathbf{v}, \mathbf{z}, \widetilde{\alpha})$ to solutions $(\widehat{\mathbf{u}}, \widehat{\mathbf{y}}, \widehat{\alpha})$ of (1.5), respectively. (We note that if in equation (1.5), we were to write $\mathbf{Y}$ instead of $\mathbf{y}_{x}$, the transformations from $\mathbf{Z}\left(=\mathbf{z}_{x}\right)$ to $\mathbf{Y}$ obtained by differentiating (1.7) and (1.10) would be more complicated, and would still involve a derivative of the function $\mathbf{Z}$ on 
their right-hand-sides.) From (1.7) we obtain

$$
2\left(\mathbf{v}_{x}-\mathbf{z}_{x}+\frac{1}{2} c_{0} \mathbf{I}+\mathbf{E}_{0}-\frac{1}{2} \mathbf{I} x\right)=(\alpha+\widetilde{\alpha})(\mathbf{z}-\mathbf{y})^{-1},
$$

and from (1.10) we get

$$
2\left(-\mathbf{v}_{x}-\mathbf{z}_{x}+\frac{1}{2} c_{0} \mathbf{I}+\mathbf{E}_{0}-\frac{1}{2} \mathbf{I} x\right)=(\widehat{\alpha}+\widetilde{\alpha})(\widehat{\mathbf{y}}-\mathbf{z})^{-1} .
$$

Setting $\widetilde{\alpha}=n$ so that $\mathbf{z}=\mathbf{z}_{n}(x), \mathbf{y}=\mathbf{z}_{n-1}(x)$ and $\widehat{\mathbf{y}}=\mathbf{z}_{n+1}(x)$ (as well as $\mathbf{v}=\mathbf{v}_{n}(x), \mathbf{u}=\mathbf{v}_{n-1}(x)$ and $\left.\widehat{\mathbf{u}}=\mathbf{v}_{n+1}(x)\right)$, these last two equations then give

$$
-2\left(2 \frac{\mathrm{d}}{\mathrm{d} x} \mathbf{z}_{n}-c_{0} \mathbf{I}-2 \mathbf{E}_{0}+\mathbf{I} x\right)=(2 n+1)\left[\mathbf{z}_{n+1}-\mathbf{z}_{n}\right]^{-1}+(2 n-1)\left[\mathbf{z}_{n}-\mathbf{z}_{n-1}\right]^{-1} .
$$

Setting now

$$
\mathbf{z}_{n}(x)=(\mathcal{E}+1) \mathbf{r}_{n}(x)+\frac{1}{2}\left(c_{0} x \mathbf{I}+2 \mathbf{E}_{0} x-\frac{1}{2} x^{2} \mathbf{I}\right),
$$

where $\mathcal{E}$ is the shift operator defined as $\mathcal{E} \mathbf{f}_{n}(x)=\mathbf{f}_{n+1}(x)$, we obtain

$$
-4 \frac{\mathrm{d}}{\mathrm{d} x} \mathbf{r}_{n}=(2 n-1)\left[\mathbf{r}_{n+1}-\mathbf{r}_{n-1}\right]^{-1}+\mathbf{a}(x)(-1)^{n},
$$

for some matrix $\mathbf{a}(x)$. Finally, the substitution

$$
\mathbf{r}_{n}(x)=\mathbf{U}_{n}(t)-\frac{1}{4} \mathbf{b}(x)(-1)^{n}, \quad t=-\frac{1}{4} x, \quad \text { where } \quad \frac{d}{d x} \mathbf{b}=\mathbf{a},
$$

yields

$$
\frac{\mathrm{d}}{\mathrm{d} t} \mathbf{U}_{n}=(2 n-1)\left(\mathbf{U}_{n+1}-\mathbf{U}_{n-1}\right)^{-1},
$$

that is, equation (1.4). We note that in the case of the scalar $P_{I I}$ equation

$$
u_{x x}=2 u^{3}+x u+\alpha
$$

the same manipulations lead to the scalar nonautonomous semidiscrete equation for $u_{n}(t)$ [6],

$$
\frac{\mathrm{d} u_{n}}{\mathrm{~d} t}=\frac{2 n-1}{u_{n+1}-u_{n-1}}
$$

Let us recall that the usual application of the ideas in $[7,8]$ to the aBTs of matrix $P_{I I}$ leads to a discrete matrix $P_{I}$ equation [1] (see also [3] for a special case), i.e., a discrete matrix equation having as a continuum limit the matrix $P_{I}$ equation

$$
\omega_{x x}=6 \omega^{2}+x \mathbf{I}+\mathbf{A},
$$

an equation given in $[4,5]$ (here $\mathbf{A}$ is an arbitrary constant matrix). This leads naturally to the question of the link between (1.18) and the matrix $P_{I}$ equation (1.21). This is one of the questions that we address in this paper. A further question addressed here is that of whether 
the matrix $P_{I}$ and $P_{I I}$ equations can be put into Hamiltonian form. It is of course well-known that this can be done for the scalar first and second Painlevé equations. We also discuss classes of solutions of the matrix $P_{I I}$ equation. We recall that the scalar second Painlevé equation has, for certain values of the parameter appearing therein, rational solutions and solutions expressible in terms of Airy functions. Here we extend these results to the matrix case. These results are also completely new. Indeed, even though the matrix $P_{I I}$ equation (1.3) was presented some twenty years ago, it is only with the work in $[1,2,3]$ that aBTs which may be used to succesively generate solutions by iterating from some initial solution were discovered for the matrix $P_{I I}$ equation.

The layout of the paper is as follows. In Section 2 we give a Hamiltonian formulation of the general $m \times m$ matrix first and second Painlevé equations. In Section 3 we begin our study of the relationship between the above semidiscrete equations and corresponding first Painlevé equations. Motivated by the derivation given in [7] of $P_{I}$ from the alternative discrete first Painlevé equation, we first consider the scalar semidiscrete equation (1.20) and show that it has as a limiting case the scalar first Painlevé equation. In Section 4 we extend our approach to the matrix case, and show how matrix $P_{I}$ can be obtained from the matrix semidiscrete equation (1.18). Also in Section 4 we consider our asymptotic analysis more generally in order to explore the sort of results that can be obtained using this approach. Section 5 is different, since we consider therein a component-wise approach to the asymptotic analysis of the matrix semidiscrete equation (1.18). In Section 6 we discuss various classes of solutions of the matrix $P_{I I}$ equation, and how they may be obtained by iteration from certain initial solutions. The paper concludes with a summary of our results and a discussion of their implications in Section 7.

\section{Hamiltonian formulations of matrix $\mathbf{P}_{I}$ and matrix $\mathbf{P}_{I I}$}

Let us begin by considering the matrix equation [1]

$$
\mathbf{w}_{x x}+3 \mathbf{w}^{2}+c_{0} \mathbf{w}+\frac{1}{2} c_{-1} \mathbf{I}+\mathbf{H}+g_{1} x \mathbf{I}=0,
$$

where $\mathbf{w}=\left(w_{i j}\right)$ is an $m \times m$ matrix of functions $w_{i j}(x), \mathbf{H}=\left(h_{i j}\right)$ is an arbitrary constant $m \times m$ matrix, and $c_{0}, c_{-1}$ and $g_{1}$ are arbitrary scalar constants. In the case $g_{1} \neq 0$, a change of independent and dependent variables allows us to transform this equation to the form (1.21). Here we do not assume $g_{1} \neq 0$. With a view to future work on the $P_{I}$ hierarchy, neither do we assume - even though we may do so without loss of generality - that $c_{0}=c_{-1}=0$. We now show that equation (2.1) can be put into Hamiltonian form, as given by the following result:

For any $m=1,2,3, \ldots$, define

$$
\begin{aligned}
\mathcal{H}_{m}\left(w_{i j}, p_{i j}, x\right)= & \frac{1}{2} \sum_{q, r=1}^{m} p_{q r} p_{r q}+\sum_{q, r, s=1}^{m} w_{q r} w_{r s} w_{s q}+\frac{1}{2} c_{0} \sum_{q, r=1}^{m} w_{q r} w_{r q}+\frac{1}{2} c_{-1} \sum_{q=1}^{m} w_{q q} \\
& +\sum_{q, r=1}^{m} h_{q r} w_{r q}+g_{1} x \sum_{q=1}^{m} w_{q q} .
\end{aligned}
$$


Then the $m \times m$ matrix equation (2.1) can be written in Hamiltonian form

$$
\frac{\mathrm{d} w_{i j}}{\mathrm{~d} x}=\frac{\partial \mathcal{H}_{m}}{\partial p_{i j}}, \quad \frac{\mathrm{d} p_{i j}}{\mathrm{~d} x}=-\frac{\partial \mathcal{H}_{m}}{\partial w_{i j}} .
$$

In order to see this, we note that (2.2) and (2.3) imply

$$
\frac{\mathrm{d}^{2} w_{i j}}{\mathrm{~d} x^{2}}=\frac{\mathrm{d}}{\mathrm{d} x}\left(\frac{\partial \mathcal{H}_{m}}{\partial p_{i j}}\right)=\frac{\mathrm{d} p_{j i}}{\mathrm{~d} x}=-\frac{\partial \mathcal{H}_{m}}{\partial w_{j i}}=-\left(3 \sum_{q=1}^{m} w_{i q} w_{q j}+c_{0} w_{i j}+\frac{1}{2} c_{-1} \delta_{i j}+h_{i j}+g_{1} x \delta_{i j}\right),
$$

so that equation (2.1) holds.

We remark that for the above Hamiltonian to be a constant of the motion we must have that

$$
\frac{\partial \mathcal{H}_{m}}{\partial x}=g_{1} \sum_{q=1}^{m} w_{q q}=0
$$

Thus, in the general case where the matrix equation (2.1) defines $m^{2}$ second order ODEs (so that $2 m^{2}$ initial conditions may be freely imposed), the above Hamiltonian is a constant of the motion if and only if $g_{1}=0$, that is, if and only if equation (2.1) is autonomous. In the scalar case with $g_{1} \neq 0$ the above results lead us (after a change of independent and dependent variables) to a well-known Hamiltonian formulation of the first Painlevé equation $\omega_{x x}=6 \omega^{2}+x$ [17].

Let us now consider the matrix equation (1.1) [1]

$$
\mathbf{u}_{x x}-2 \mathbf{u}^{3}+c_{0} \mathbf{u}+\mathbf{u} \mathbf{E}_{0}+\mathbf{E}_{0} \mathbf{u}+2 g_{0} x \mathbf{u}-\alpha \mathbf{I}=\mathbf{0} .
$$

As noted earlier, we prefer not to absorb the term $c_{0} \mathbf{u}$ in the terms $\mathbf{u} \mathbf{E}_{0}+\mathbf{E}_{0} \mathbf{u}$. Neither do we assume here that $g_{0} \neq 0$. We now show that equation (2.6) can be put into Hamiltonian form. We give the following result:

For any $m=1,2,3, \ldots$, define

$$
\begin{aligned}
\mathcal{K}_{m}\left(u_{i j}, p_{i j}, x\right)= & \frac{1}{2} \sum_{q, r=1}^{m} p_{q r} p_{r q}-\frac{1}{2} \sum_{q, r, s, t=1}^{m} u_{q r} u_{r s} u_{s t} u_{t q}+\frac{1}{2} c_{0} \sum_{q, r=1}^{m} u_{q r} u_{r q}+\sum_{q, r, s=1}^{m} u_{q r} e_{r s} u_{s q} \\
& +g_{0} x \sum_{q, r=1}^{m} u_{q r} u_{r q}-\alpha \sum_{q=1}^{m} u_{q q} .
\end{aligned}
$$

Then the $m \times m$ matrix equation (2.6) can be written in Hamiltonian form

$$
\frac{\mathrm{d} u_{i j}}{\mathrm{~d} x}=\frac{\partial \mathcal{K}_{m}}{\partial p_{i j}}, \quad \frac{\mathrm{d} p_{i j}}{\mathrm{~d} x}=-\frac{\partial \mathcal{K}_{m}}{\partial u_{i j}} .
$$


In order to see this, we note that (2.7) and (2.8) imply

$$
\begin{aligned}
\frac{\mathrm{d}^{2} u_{i j}}{\mathrm{~d} x^{2}} & =\frac{\mathrm{d}}{\mathrm{d} x}\left(\frac{\partial \mathcal{K}_{m}}{\partial p_{i j}}\right)=\frac{\mathrm{d} p_{j i}}{\mathrm{~d} x}=-\frac{\partial \mathcal{K}_{m}}{\partial u_{j i}} \\
& =-\left(-2 \sum_{q, r=1}^{m} u_{i q} u_{q r} u_{r j}+c_{0} u_{i j}+\sum_{q=1}^{m}\left(u_{i q} e_{q j}+e_{i q} u_{q j}\right)+2 g_{0} x u_{i j}-\alpha \delta_{i j}\right),
\end{aligned}
$$

so that equation (2.6) holds.

We remark that for the above Hamiltonian to be a constant of the motion we must have that

$$
\frac{\partial \mathcal{K}_{m}}{\partial x}=g_{0} \sum_{q, r=1}^{m} u_{q r} u_{r q}=0 .
$$

Thus, in the general case where the matrix equation (2.6) defines $m^{2}$ second order ODEs (i.e., such that $2 m^{2}$ initial conditions may be freely imposed), the above Hamiltonian is a constant of the motion if and only if $g_{0}=0$, i.e., if and only if equation (2.6) is autonomous. In the scalar case with $g_{0} \neq 0$ the above results lead us (after a change of independent and dependent variables) to a well-known Hamiltonian formulation of the second Painlevé equation $u_{x x}=$ $2 x^{3}+x u+\alpha[17]$.

\section{The scalar case}

We begin our asymptotic analysis of semidiscrete equations by considering first of all the scalar equation (1.20) [6], that is

$$
\frac{\mathrm{d} u_{n}}{\mathrm{~d} t}\left(u_{n+1}-u_{n-1}\right)=2 n-1 .
$$

We define

$$
u_{n}(t)=\sqrt{2} \widehat{u}(x, t), \quad \text { where } \quad x=n-\frac{1}{2},
$$

where this variable $x$ is different from that used in Sections 1 and 2; and we immediately neglect the hats so that

$$
x=u_{t}(x, t) \Delta u(x, t), \quad \text { where } \quad \Delta f(x)=f(x+1)-f(x-1) .
$$

Whilst $u=x \sqrt{t}$ and $u=\frac{1}{4} x^{2}+t$ are solutions of equation (3.1), we consider the dynamics in the far-field where $x \gg 1$. Hence we introduce the small parameter $h \ll 1$ and new space and time variables, $y, \tau$ via the substitution

$$
x=\frac{a}{h^{1+\alpha}}+\frac{y}{h}, \quad t=h^{-\sigma} \tau, \quad u(x, t)=h^{\delta} u_{0}(y, \tau)+h^{\delta+\beta} u_{1}(y, \tau)+h^{\delta+2 \beta} u_{2}(y, \tau),
$$

with $\alpha \geq 0, \beta>0$. This corresponds to an expansion in the far field region $\left(x=a h^{-1-\alpha}\right)$ with the new independent variable $(y)$ ranging over many lattice sites: when $x$ is increased by one, 
$y$ only increases by a little $(O(h))$; in order for $y$ to be increased by an $O(1)$ amount, $x$ needs to increase by $O\left(h^{-1}\right)$.

Substituting the expressions (3.4) into (3.3) leads to

$$
\begin{aligned}
\frac{a}{h^{\alpha+1}}+\frac{y}{h}= & h^{2 \delta+\sigma+1}\left(u_{0, \tau}+h^{\beta} u_{1, \tau}+h^{2 \beta} u_{2, \tau}\right) \times \\
& \left(2 u_{0, y}+2 h^{\beta} u_{1, y}+2 h^{2 \beta} u_{2, y}+\frac{h^{2} u_{0, y y y}}{3}+\frac{h^{\beta+2} u_{1, y y y}}{3}+\frac{h^{4} u_{0, y y y y y}}{60}\right) .
\end{aligned}
$$

The leading order terms on each side balance when $2 \delta+\sigma+1=-1-\alpha$ leading to the equation

$$
a=2 u_{0, y} u_{0, \tau}
$$

for $u_{0}(y, \tau)$, which is solved by

$$
u_{0}=k y+\frac{a \tau}{2 k} \text {. }
$$

We choose $\beta=2, \alpha=4, \delta=0$ so that $\sigma=-6 ; \beta=2$ is required for the third derivative term obtained from $h^{\beta+2} u_{0, \tau} u_{1, y y y}$ to balance the quadratic first derivative terms $h^{2 \beta} u_{1, \tau} u_{1, y}$. For the magnitude of the displacements $u$ in (3.4) to be $O(1)$, we have $\delta=0$ and the timescale for the evolution of such displacements is rather fast, being given by $t=h^{6} \tau(\sigma=-6)$. This choice of asymptotic scaling is also used for the matrix problem considered later - see the text following equation (4.3), as well as the component-wise analysis following equations (5.6)-(5.7). The governing equations below would be generated by other choices for $\delta, \sigma$ : for example, if we choose $\sigma=0$ and $\delta=-3$, then we find large amplitude disturbances $\left(u=O\left(h^{-3}\right)\right)$ which evolve on the standard timescale $\tau=O(1)$.

The equations obtained at the next two orders of $h$ in (3.5) are

$$
\begin{aligned}
& 0=2 u_{0, y} u_{1, \tau}+2 u_{0, \tau} u_{1, y}+\frac{1}{3} u_{0, \tau} u_{0, y y y}, \\
& y=2 u_{0, y} u_{2, \tau}+2 u_{1, \tau} u_{1, y}+\frac{1}{3} u_{1, \tau} u_{0, y y y}+\frac{1}{3} u_{0, \tau} u_{1, y y y}+2 u_{0, \tau} u_{2, y}+\frac{1}{60} u_{0, \tau} u_{0, y y y y y} .
\end{aligned}
$$

With the solution (3.7), equation (3.8) is a first-order travelling wave equation

$$
0=2 k u_{1, \tau}+\frac{a}{k} u_{1, y}
$$

which implies

$$
u_{1}(y, \tau)=u_{1}(z), \quad z=y-\frac{a \tau}{2 k^{2}} .
$$

Noting the solution (3.7) for $u_{0}$, eq. (3.9) becomes

$$
y=2 k u_{2, \tau}+2 u_{1, \tau} u_{1, y}+\frac{a}{6 k} u_{1, y y y}+\frac{a}{k} u_{2, y} .
$$

Transforming this into the travelling wave variables $u_{1}=u_{1}(z), u_{2}=u_{2}(z, \tau)$ yields

$$
z+\frac{a \tau}{2 k^{2}}=\frac{a u_{1, z z z}}{6 k}+2 k u_{2, \tau}-\frac{a}{k^{2}} u_{1, z}^{2}
$$


We note that the terms involving $u_{2, z}$ cancel, leaving just the $u_{2, \tau}$ term. If we now take $u_{2}=$ $a \tau^{2} / 8 k^{3}$ and define $f(z)$ as $f(z)=u_{1, z}(z)$, we obtain the equation

$$
\frac{6 k^{2} z}{a}=k \frac{\mathrm{d}^{2} f}{\mathrm{~d} z^{2}}-6 f(z)^{2},
$$

which is $P_{I}$ in $f(z)$. As remarked earlier, this derivation of $P_{I}$ from equation (3.1) is motivated by the derivation given in [7] of $P_{I}$ from the alternative discrete first Painlevé equation. We now turn to the matrix form of (3.1).

\section{The matrix case}

The matrix form of (3.1) is equation (1.4) [6], which is

$$
\frac{\mathrm{d}}{\mathrm{d} t} \mathbf{U}_{n}=(2 n-1)\left(\mathbf{U}_{n+1}-\mathbf{U}_{n-1}\right)^{-1},
$$

where $\mathbf{U}_{n}$ is a square $(m \times m)$ matrix. This we rewrite as

$$
\mathbf{U}_{t}=\frac{\mathrm{d}}{\mathrm{d} t} \mathbf{U}=x(\Delta \mathbf{U})^{-1},
$$

using a change of variables analogous to (3.2). We analyse this equation in two ways: firstly by considering the matrix form, to find connections between this and the matrix $P_{I}$ equation; and secondly in Section 5 by taking example matrices and analysing the coupled equations component by component. In both approaches, we focus on the dynamics in the far-field asymptotics.

\subsection{Asymptotic analysis of the matrix equation}

We use the scalings given in (3.4) with $\alpha=4, \beta=2, \delta=0, \sigma=-6$, together with an asymptotic ansatz for the series expansion of the solution as

$$
\mathbf{U}(x, t)=\mathbf{U}_{0}(x, t)+h^{2} \mathbf{U}_{1}(x, t)+h^{4} \mathbf{U}_{2}(x, t)+\ldots,
$$

to express the matrix problem $x \mathbf{I}=\mathbf{U}_{t} \Delta \mathbf{U}$ as

$$
\begin{aligned}
& a h^{-5} \mathbf{I}+y h^{-1} \mathbf{I}=h^{-5}\left(\mathbf{U}_{0, \tau}+h^{2} \mathbf{U}_{1, \tau}+h^{4} \mathbf{U}_{2, \tau}\right)\left(2 \mathbf{U}_{0, y}+2 h^{2} \mathbf{U}_{1, y}+2 h^{4} \mathbf{U}_{2, y}\right. \\
& \left.+\frac{1}{3} h^{2} \mathbf{U}_{0, y y y}+\frac{1}{3} h^{4} \mathbf{U}_{1, y y y}+\frac{1}{60} h^{4} \mathbf{U}_{0, y y y y y}\right) \\
& =2 h^{-5} \mathbf{U}_{0, \tau} \mathbf{U}_{0, y}+h^{-3}\left(2 \mathbf{U}_{1, \tau} \mathbf{U}_{0, y}+2 \mathbf{U}_{0, \tau} \mathbf{U}_{1, y}+\frac{1}{3} \mathbf{U}_{0, \tau} \mathbf{U}_{0, y y y}\right) \\
& +h^{-1}\left(2 \mathbf{U}_{0, \tau} \mathbf{U}_{2, y}+2 \mathbf{U}_{1, \tau} \mathbf{U}_{1, y}+2 \mathbf{U}_{2, \tau} \mathbf{U}_{0, y}+\frac{1}{3} \mathbf{U}_{0, \tau} \mathbf{U}_{1, y y y}\right. \\
& \left.+\frac{1}{3} \mathbf{U}_{1, \tau} \mathbf{U}_{0, y y y}+\frac{1}{60} \mathbf{U}_{0, \tau} \mathbf{U}_{0, y y y y y}\right)+O(h) \text {. }
\end{aligned}
$$

The leading order expression comes from terms of $O\left(h^{-5}\right)$ from (4.4), namely

$$
a \mathbf{I}=2 \mathbf{U}_{0, \tau} \mathbf{U}_{0, y}
$$


which has the solution

$$
\mathbf{U}_{0}=y \mathbf{K}+\frac{1}{2} a \tau \mathbf{K}^{-1}
$$

for any arbitrary nonsingular constant matrix $\mathbf{K}$.

At next order, that is, $O\left(h^{-3}\right)$, we have

$$
\mathbf{0}=\mathbf{U}_{1, \tau} \mathbf{U}_{0, y}+\mathbf{U}_{0, \tau} \mathbf{U}_{1, y}
$$

which, using (4.6), implies

$$
\mathbf{0}=2 \mathbf{K U}_{1, \tau} \mathbf{K}+a \mathbf{U}_{1, y} .
$$

Imposing the travelling wave ansatz $\mathbf{U}_{1}=\mathbf{U}_{1}(z)$ with $z=y-c \tau$ in this equation leads to

$$
\mathbf{K U}_{1, z} \mathbf{K}=\gamma \mathbf{U}_{1, z}, \quad \gamma=\frac{a}{2 c},
$$

which is a deceptively simple equation for $\mathbf{U}_{1, z}$ given $\mathbf{K}$.

In the next subsection, we consider a special case of $\mathbf{K}$ which simplifies the solution of (4.9) and allows us to derive the general case of the matrix $P_{I}$ equation in $\mathbf{U}_{1, z}$. In Section 4.3.1 we return to a more general form of $\mathbf{K}$, which leads to a restricted equation for $\mathbf{U}_{1, z}$.

\subsection{Derivation of the matrix $\mathbf{P}_{I}$ equation}

Here, we consider the particular form of (4.6) with $\mathbf{K}$ being a multiple of the identity matrix, that is, $\mathbf{K}=k \mathbf{I}$ so that

$$
\mathbf{U}_{0}=\left(k y+\frac{a \tau}{2 k}\right) \mathbf{I}
$$

This solution simplifies later calculations, since $\mathbf{U}_{0}$, in addition to satisfying $\mathbf{U}_{0, y y y}=0=\mathbf{U}_{0, y y y y y}$, also commutes with all other matrices.

At $O\left(h^{-3}\right)$, from which (4.9) is derived, we now have

$$
k^{2} \mathbf{U}_{1, z}=\gamma \mathbf{U}_{1, z}
$$

hence $c=a / 2 \gamma=a / 2 k^{2}$. Whilst this calculation provides the speed $(c)$ of the solution, the shape of the wave remains undetermined provided this specific value of $c$ is chosen; there are no conditions on the elements of $\mathbf{U}_{1, z}$ from (4.11), and, in particular, no restriction on the number of nonzero elements. Therefore, we need to proceed to higher order terms to determine an equation for the profile $\mathbf{U}_{1}$.

At $O\left(h^{-1}\right)$, after substituting for $\mathbf{U}_{0}$, we have

$$
y \mathbf{I}=\frac{a}{k} \mathbf{U}_{2, y}+2 \mathbf{U}_{1, \tau} \mathbf{U}_{1, y}+2 k \mathbf{U}_{2, \tau}+\frac{a}{6 k} \mathbf{U}_{1, y y y} .
$$

Transforming this equation into the moving coordinate frame $(z, \tau)$, and setting $\mathbf{U}_{1}=\mathbf{U}_{1}(z)$ with $\mathbf{U}_{2}=\mathbf{U}_{2}(z, \tau)$, we obtain

$$
z \mathbf{I}+\frac{a \tau}{2 k^{2}} \mathbf{I}=2 k \mathbf{U}_{2, \tau}-\frac{a}{k^{2}} \mathbf{U}_{1, z}^{2}+\frac{a}{6 k} \mathbf{U}_{1, z z z}
$$


(again the terms in $\mathbf{U}_{2, z}$ cancel). We then choose

$$
\mathbf{U}_{2}(z, \tau)=\frac{a \tau^{2}}{8 k^{3}} \mathbf{I}+\tau \mathbf{B}+\mathbf{C},
$$

where $\mathbf{B}$ and $\mathbf{C}$ are arbitrary constant matrices. The matrix $\mathbf{C}$ can be neglected; however, the introduction of the arbitrary matrix $\mathbf{B}$ into the ensuing equations is significant. With the above choice of $\mathbf{U}_{2}$, and setting $\mathbf{F}=\mathbf{U}_{1, z}$, we obtain from (4.13) the equation

$$
z \mathbf{I}=\frac{a}{6 k} \mathbf{F}_{z z}-\frac{a}{k^{2}} \mathbf{F}^{2}+2 k \mathbf{B},
$$

which is the matrix form of $P_{I}$. If the size of these matrices is given as $m \times m$, then (4.15), constitutes a set of $m^{2}$ coupled second-order ODES. We remark that in the scalar case, the inclusion of additive terms $b \tau+c$ in $u_{2}$ does not lead to a generalized equation, since an additional constant term in (3.14) can be removed using a shift on $z$.

Whilst the above derivation started from $\mathbf{U}_{t}(\Delta \mathbf{U})=x \mathbf{I}$, we note that (4.2) is also equivalent to $x \mathbf{I}=(\Delta \mathbf{U}) \mathbf{U}_{t}$. A similar derivation from this latter equation also yields (4.15).

\subsection{Asymptotic analysis: further considerations}

\subsubsection{Second order terms}

We continue with our asymptotic analysis but with a more general form for $\mathbf{K}$ in equations (4.6) and (4.9). In order to illustrate the kind of results that may be obtained, let us consider the construction of a solution $\mathbf{U}_{1, z}$ of (4.9) in the case where $\mathbf{K}$ has a full set of distinct eigenvalues $\left\{\lambda_{j}\right\}$ and so is diagonalisable; we write $\mathbf{K}=\mathbf{P D P}^{-1}$ with $\mathbf{D}=\operatorname{diag}\left\{\lambda_{j}\right\}$. We also introduce $\mathbf{Q}=\mathbf{P}^{-1} \mathbf{U}_{1, z} \mathbf{P}$ so that $\mathbf{U}_{1, z}=\mathbf{P Q P} \mathbf{P}^{-1}$. Hence the problem (4.9) can be written as: given $\mathbf{D}$, find $\mathbf{Q}$ such that

$$
\mathbf{D Q D}=\gamma \mathbf{Q}
$$

This problem is similar to the previously studied discrete Lyapunov equation $[18,19]$.

We solve this by considering the effect of each side on the unit vectors $\mathbf{e}_{j}$. Since $\mathbf{D}$ is diagonal, these unit vectors are eigenvectors with corresponding eigenvalues $\lambda_{j}$ : De $\mathbf{e}_{j}=\lambda_{j} \mathbf{e}_{j}$. It follows from (4.16) that $\mathbf{Q} \mathbf{e}_{j}$ (if nonzero) is also an eigenvector of $\mathbf{D}$ since we have

$$
\lambda_{j} \mathbf{D}\left(\mathbf{Q} \mathbf{e}_{j}\right)=\gamma\left(\mathbf{Q} \mathbf{e}_{j}\right)
$$

and the corresponding eigenvalue is $\gamma / \lambda_{j}$. There are two cases to consider, depending on $\mathbf{Q} \mathbf{e}_{j}$, namely

- $\mathbf{Q} \mathbf{e}_{j}=f_{j}(z) \mathbf{e}_{j}$, then $\gamma=\lambda_{j}^{2}$ so $c=a / 2 \gamma=a /\left(2 \lambda_{j}^{2}\right)$. This case provides diagonal elements to the matrix $\mathbf{Q}$.

- $\mathbf{Q} \mathbf{e}_{j}=f_{k}(z) \mathbf{e}_{k}$ with $k \neq j$, then $\lambda_{j} \lambda_{k}=\gamma$ so that $c=a / 2 \gamma=a /\left(2 \lambda_{j} \lambda_{k}\right)$. This case provides off-diagonal elements to the matrix $\mathbf{Q}$. 
(Of course, the same choice of $\gamma$ has to be obtained, a condition which may then restrict the possible combinations of the above cases.) We see from this construction that in the case of $m \times m$ matrices, $\mathbf{Q}$ will depend on $m$ functions $f_{j}(z)$. We also note in passing that consideration of the case where $\mathbf{Q} \mathbf{e}_{j}=\mathbf{0}$ for some $j$, that is, where one column of $\mathbf{Q}$ is entirely composed of zeros - and (4.17) is automatically satisfied with $\gamma$ undetermined - leads to an inconsistency, as explained in Section 4.3.3.

We now consider two $2 \times 2$ examples, one to illustrate each of the above two cases.

\subsubsection{Examples in the $\mathbf{2} \times \mathbf{2}$ case}

Case 1: $\mathbf{Q} \mathbf{e}_{j}=f_{j}(z) \mathbf{e}_{j}$

To illustrate Case 1 , where $\gamma=\lambda_{1}^{2}=\lambda_{2}^{2}$, we take $\gamma=1$ with $\lambda_{1}=+1, \lambda_{2}=-1$, and

$$
\mathbf{K}=\left(\begin{array}{cc}
7 & -4 \\
12 & -7
\end{array}\right), \quad \mathbf{D}=\left(\begin{array}{cc}
1 & 0 \\
0 & -1
\end{array}\right), \quad \mathbf{P}=\left(\begin{array}{ll}
2 & 1 \\
3 & 2
\end{array}\right), \quad \mathbf{P}^{-1}=\left(\begin{array}{cc}
2 & -1 \\
-3 & 2
\end{array}\right)
$$

so that the solution is given by

$$
\mathbf{Q}=\left(\begin{array}{cc}
f_{1}(z) & 0 \\
0 & f_{2}(z)
\end{array}\right), \quad \mathbf{U}_{1, z}=\left(\begin{array}{cc}
4 f_{1}-3 f_{2} & -2 f_{1}+2 f_{2} \\
6 f_{1}-6 f_{2} & -3 f_{1}+4 f_{2}
\end{array}\right)=f_{1}\left(\begin{array}{cc}
4 & -2 \\
6 & -3
\end{array}\right)+f_{2}\left(\begin{array}{ll}
-3 & 2 \\
-6 & 4
\end{array}\right) .
$$

Even though the $2 \times 2$ matrix $\mathbf{U}_{1, z}$ has four elements, the solution has only two degrees of freedom, $f_{1}(z), f_{2}(z)$. Consideration of higher order terms may impose further constraints on the system of equations for $f_{1}(z), f_{2}(z)$. In this example, $\mathbf{Q}$ has nonzero elements only on the leading diagonal.

Case 2: $\mathbf{Q} \mathbf{e}_{j}=f_{k}(z) \mathbf{e}_{k}$ with $j \neq k$

For Case 2, where $\gamma=\lambda_{1} \lambda_{2}$, we take $\gamma=2$ with $\lambda_{1}=1, \lambda_{2}=2$ and

$$
\mathbf{K}=\left(\begin{array}{ll}
-2 & 2 \\
-6 & 5
\end{array}\right), \quad \mathbf{D}=\left(\begin{array}{ll}
1 & 0 \\
0 & 2
\end{array}\right), \quad \mathbf{P}=\left(\begin{array}{ll}
2 & 1 \\
3 & 2
\end{array}\right), \quad \mathbf{P}^{-1}=\left(\begin{array}{cc}
2 & -1 \\
-3 & 2
\end{array}\right)
$$

so that the solution is given by

$$
\mathbf{Q}=\left(\begin{array}{cc}
0 & f_{1}(z) \\
f_{2}(z) & 0
\end{array}\right), \quad \mathbf{U}_{1, z}=\left(\begin{array}{cc}
-6 f_{1}+2 f_{2} & 4 f_{1}-f_{2} \\
-9 f_{1}+4 f_{2} & 6 f_{1}-2 f_{2}
\end{array}\right)=f_{1}\left(\begin{array}{cc}
-6 & 4 \\
-9 & 6
\end{array}\right)+f_{2}\left(\begin{array}{cc}
2 & -1 \\
4 & -2
\end{array}\right)
$$

As in the previous example, the solution derived thus far has only two degrees of freedom, $f_{1}(z), f_{2}(z)$, rather than the four which may be expected from a $2 \times 2$ matrix problem, and further constraints may be imposed on the system when we consider higher order terms. In this case, $\mathbf{Q}$ has nonzero elements only in off-diagonal locations and only one nonzero element in each column. 


\subsubsection{Third order terms}

At $O\left(h^{-1}\right)$, which is the final order that we consider, we obtain from (4.4) the equation

$$
y \mathbf{I}=2 \mathbf{U}_{0, \tau} \mathbf{U}_{2, y}+2 \mathbf{U}_{1, \tau} \mathbf{U}_{1, y}+2 \mathbf{U}_{2, \tau} \mathbf{U}_{0, y}+\frac{1}{3} \mathbf{U}_{0, \tau} \mathbf{U}_{1, y y y}+\frac{1}{3} \mathbf{U}_{1, \tau} \mathbf{U}_{0, y y y}+\frac{1}{60} \mathbf{U}_{0, \tau} \mathbf{U}_{0, y y y y y},
$$

which, substituting from (4.6), simplifies to

$$
y \mathbf{K}=a \mathbf{U}_{2, y}+2 \mathbf{K U}_{1, \tau} \mathbf{U}_{1, y}+2 \mathbf{K U}_{2, \tau} \mathbf{K}+\frac{1}{6} a \mathbf{U}_{1, y y y} .
$$

Now transforming to travelling wave coordinates, $\mathbf{U}_{1}=\mathbf{U}_{1}(z)$ and $\mathbf{U}_{2}=\mathbf{U}_{2}(z, \tau)$, yields

$$
z \mathbf{K}+c \tau \mathbf{K}=a \mathbf{U}_{2, z}-2 c \mathbf{K U}_{1, z}^{2}+2 \mathbf{K}\left(\mathbf{U}_{2, \tau}-c \mathbf{U}_{2, z}\right) \mathbf{K}+\frac{1}{6} a \mathbf{U}_{1, z z z},
$$

(we note that in this case the terms in $\mathbf{U}_{2, z}$ do not cancel). However, if we now choose

$$
\mathbf{U}_{2}=\frac{1}{4} c \tau^{2} \mathbf{K}^{-1}+\tau \mathbf{B}+\mathbf{C}
$$

where $\mathbf{B}$ and $\mathbf{C}$ are arbitrary constant matrices, then we obtain (noting that $\mathbf{U}_{2, z}=\mathbf{0}$ )

$$
z \mathbf{I}=\frac{1}{6} a \mathbf{K}^{-1} \mathbf{U}_{1, z z z}-2 c \mathbf{U}_{1, z}^{2}+2 \mathbf{B K}
$$

which is equivalent to

$$
z \mathbf{I}=\frac{1}{6} a \mathbf{D}^{-1} \mathbf{Q}_{z z}-2 c \mathbf{Q}^{2}+2 \widetilde{\mathbf{B}} \mathbf{D}
$$

where $\widetilde{\mathbf{B}}=\mathbf{P}^{-1} \mathbf{B P}$ and, as above, $\mathbf{U}_{1, z}=\mathbf{P Q P} \mathbf{P}^{-1}$. This equation, together with (4.26) are both variants of the matrix $P_{I}$ equation. We note, as mentioned earlier, that the case where $\mathbf{Q}$ has a column (say, the $j$ th column) entirely composed of zeros leads to an inconsistency, since the $j$ th column of $\mathbf{Q}^{2}$ will also be entirely composed of zeros and the $(j, j)$-element of equation (4.27) then gives $z=$ const.

In Case 1 of $\S 4.3 .2$, we note from (4.18)-(4.19) that $\mathbf{D}, \mathbf{Q}, \mathbf{I}$ are diagonal, and so $\widetilde{\mathbf{B}}$ in (4.27) should also be diagonal. Thus in this case the components of the matrix $P_{I}$ equations (4.26) and (4.27) decouple into two copies of the scalar $P_{I}$ equation.

Case 2 of $\$ 4.3 .2$ differs. Whilst (4.20) -(4.21) result in $\mathbf{D}, \mathbf{Q}^{2}$, $\mathbf{I}$ being diagonal, $\mathbf{Q}$ is not. Thus the off-diagonal elements of (4.27) result in equations $f_{j}^{\prime \prime}(z)=$ const, $j=1,2$, and the diagonal elements result in $-2 c f_{1}(z) f_{2}(z)=z+$ const. These equations result in the solutions $f_{j}(z)=\alpha_{j} z+\beta_{j}, j=1,2$, subject to $\alpha_{1} \alpha_{2}=0$ and with $\widetilde{\mathbf{B}}$ in (4.27) chosen appropriately.

If, instead of considering the far field asymptotics of $x \mathbf{I}=\mathbf{U}_{t}(\Delta \mathbf{U})$, which led to (4.26)(4.27), we performed a similar analysis on $x \mathbf{I}=(\Delta \mathbf{U}) \mathbf{U}_{t}$, then the outcome differs only slightly. We still obtain the equation (4.16) from the second order terms, but in place of (4.26) we obtain

$$
z \mathbf{I}=\frac{1}{6} a \mathbf{U}_{1, z z z} \mathbf{K}^{-1}-2 c \mathbf{U}_{1, z}^{2}+2 \mathbf{K B}
$$

which is equivalent to

$$
z \mathbf{I}=\frac{1}{6} a \mathbf{Q}_{z z} \mathbf{D}^{-1}-2 c \mathbf{Q}^{2}+2 \mathbf{D} \widetilde{\mathbf{B}}
$$


where $\mathbf{D}=\mathbf{P}^{-1} \mathbf{K P}, \mathbf{Q}=\mathbf{P}^{-1} \mathbf{U}_{1, z} \mathbf{P}$, and $\widetilde{\mathbf{B}}=\mathbf{P}^{-1} \mathbf{B P}$. In contrast with section 4.2, where the special leading order solution (4.10) results in the two originating equations $x \mathbf{I}=\mathbf{U}_{t}(\Delta \mathbf{U})$ and $x \mathbf{I}=(\Delta \mathbf{U}) \mathbf{U}_{t}$ both leading to the full matrix first Painlevé equation (4.15), the more general ansatz (4.6) for $\mathbf{U}_{0}$ leads to apparently different matrix first Painlevé equations given by the pairs (4.26)-(4.27) and (4.28)-(4.29). We refer to (4.15) as the full matrix first Painlevé equation since there are no restrictions on $\mathbf{F}$ or $\mathbf{B}$.

In the next section we consider the asymptotic reduction in more detail, taking a componentby-component approach to allow the exploitation of special properties of the system.

\section{Component-wise analysis}

Instead of considering the matrix $\mathbf{U}$ as a single entity, we take a particular example of a $2 \times 2$ system and consider each component. The matrix equation (4.2) implies both

$$
(\Delta \mathbf{U})\left(\mathbf{U}_{t}\right)=x \mathbf{I}, \quad \text { and } \quad\left(\mathbf{U}_{t}\right)(\Delta \mathbf{U})=x \mathbf{I}
$$

Taking the difference of these two equations implies that the commutator, $\left[\mathbf{U}_{t}, \Delta \mathbf{U}\right]=\mathbf{0}$, where $[M, N]=M N-N M$. One class of $2 \times 2$ matrices which enjoys the property that all elements commute is the set of matrices of the form

$$
\mathbf{M}=\left(\begin{array}{cc}
a & \lambda(a-d) \\
\mu(a-d) & d
\end{array}\right), \quad \mathbf{N}=\left(\begin{array}{cc}
p & \lambda(p-q) \\
\mu(p-q) & q
\end{array}\right)
$$

with the same $\lambda, \mu$ in both matrices, and $a, d, p, q$ all being arbitrary. To illustrate a particular example, we take, for simplicity, $\lambda=\mu=\frac{1}{2}, a, p=u+v, d, q=u-v$, so that the matrix $\mathbf{U}$ is given by

$$
\mathbf{U}(x, t)=\left(\begin{array}{cc}
u(x, t)+v(x, t) & v(x, t) \\
v(x, t) & u(x, t)-v(x, t)
\end{array}\right) .
$$

Using the latter equation in the second equation of (5.1), we obtain

$$
x \mathbf{I}=\left(\begin{array}{cc}
u_{t}+v_{t} & v_{t} \\
v_{t} & u_{t}-v_{t}
\end{array}\right)\left(\begin{array}{cc}
\Delta u+\Delta v & \Delta v \\
\Delta v & \Delta u-\Delta v
\end{array}\right) .
$$

Although this constitutes a system of four equations, there are only two independent equations contained in (5.4), which can be expressed as

$$
x=u_{t} \Delta u+2 v_{t} \Delta v, \quad 0=u_{t} \Delta v+v_{t} \Delta u .
$$

These can be obtained by taking the sum and difference of the equations on the leading diagonal.

In section 5.1 we analyse nontrivial solutions of this coupled system. There are two trivial solutions which we note here and do not mention again: (i) $v=0, u \neq 0$, which leads to a diagonal matrix for $\mathbf{U}$ which is simply $\mathbf{U}=u(x, t) \mathbf{I}$, and which returns us to the scalar case; (ii) $u=0$ and $v \neq 0$, which again leads to an equation similar to the scalar case but now with $\mathbf{U}$ non-diagonal. In the following analysis, we assume $u \neq 0 \neq v$. 


\subsection{Far-field asymptotics for the $(\boldsymbol{u}, \boldsymbol{v})$ system}

Following the derivation of $P_{I}$ from the scalar difference equation in Section 3, we pursue a similar asymptotic analysis of the independent component equations (5.5) of the matrix equation (5.4),correponding to a particular $2 \times 2$ system for the choice (5.3). There is a degree of arbitrariness in the scaling of time and the magnitudes of $u, v$. Provided $2 \delta+\sigma+6=0$, the leading order balance is non-trivial and the following analysis holds. We follow the scalings (3.4) and introduce

$$
x=\frac{a}{h^{5}}+\frac{y}{h}, \quad t=h^{6} \tau,
$$

where $a$ is a constant, $y, \tau$ are the new independent variables, describing the rapid kinetics in the far field, where the dependent variables are assumed to be $O(1)$ and are expanded in the asymptotic series

$$
u(x, t)=u_{0}(y, \tau)+h^{2} u_{1}(y, \tau)+h^{4} u_{2}(y, \tau), \quad v(x, t)=v_{0}(y, \tau)+h^{2} v_{1}(y, \tau)+h^{4} v_{2}(y, \tau) .
$$

Keeping terms of the three largest magnitudes, (5.5) yields

$$
\begin{aligned}
\frac{a}{h^{5}}+\frac{y}{h}= & \frac{2}{h^{5}}\left[\left(u_{0, \tau}+h^{2} u_{1, \tau}+h^{4} u_{2, \tau}\right)\left(u_{0, y}+h^{2} u_{1, y}+h^{4} u_{2, y}+\frac{1}{6} h^{2} u_{0, y y y}+\frac{1}{6} h^{4} u_{1, y y y}+\frac{1}{120} h^{4} u_{0, y y y y y}\right)\right. \\
& \left.+2\left(v_{0, \tau}+h^{2} v_{1, \tau}+h^{4} v_{2, \tau}\right)\left(v_{0, y}+h^{2} v_{1, y}+h^{4} v_{2, y}+\frac{1}{6} h^{2} v_{0, y y y}+\frac{1}{6} h^{4} v_{1, y y y}+\frac{1}{120} h^{4} v_{0, y y y y y}\right)\right], \\
0= & \frac{2}{h^{5}}\left[\left(u_{0, \tau}+h^{2} u_{1, \tau}+h^{4} u_{2, \tau}\right)\left(v_{0, y}+h^{2} v_{1, y}+h^{4} v_{2, y}+\frac{1}{6} h^{2} v_{0, y y y}+\frac{1}{6} h^{4} v_{1, y y y}+\frac{1}{120} h^{4} v_{0, y y y y y}\right)\right. \\
& \left.+\left(v_{0, \tau}+h^{2} v_{1, \tau}+h^{4} v_{2, \tau}\right)\left(u_{0, y}+h^{2} u_{1, y}+h^{4} u_{2, y}+\frac{1}{6} h^{2} u_{0, y y y}+\frac{1}{6} h^{4} u_{1, y y y}+\frac{1}{120} h^{4} u_{0, y y y y y}\right)\right] .
\end{aligned}
$$

We now expand these expressions and consider terms at each power of $h$ in turn.

Terms of $O\left(h^{-5}\right)$ in (5.8) are leading order and imply

$$
a=2 u_{0, \tau} u_{0, y}+4 v_{0, \tau} v_{0, y}, \quad 0=u_{0, \tau} v_{0, y}+v_{0, \tau} u_{0, y},
$$

which can be solved by the linear functions

$$
u_{0}=k(y+\theta \tau), \quad v_{0}=k \phi(y-\theta \tau), \quad \theta=\frac{a}{2 k^{2}\left(1-2 \phi^{2}\right)},
$$

where $k, \phi$ are arbitrary constants, with $\phi \neq \pm 1 / \sqrt{2}$, and $\theta$ is given by (5.10). We remark that in terms of the matrix derivation discussed in Section 4 , we have

$$
\mathbf{K}=k\left(\begin{array}{cc}
1+\phi & \phi \\
\phi & 1-\phi
\end{array}\right)
$$

which is singular only if $\phi= \pm 1 / \sqrt{2}$. Thus the existence of a solution of the form (4.6) is related to the existence of a solution here of the form (5.10). 


\subsection{Second order terms}

At the next order, namely $O\left(h^{-3}\right)$, equation (5.8) provides the equations

$$
\begin{aligned}
& 0=2 u_{1, \tau} u_{0, y}+4 v_{1, \tau} v_{0, y}+2 u_{0, \tau} u_{1, y}+4 v_{0, \tau} v_{1, y}+\frac{1}{3} u_{0, \tau} u_{0, y y y}+\frac{2}{3} v_{0, \tau} v_{0 y y y}, \\
& 0=2 u_{1, \tau} v_{0, y}+2 v_{1, \tau} u_{0, y}+2 u_{0, \tau} v_{1, y}+2 v_{0, \tau} u_{1, y}+\frac{1}{3} u_{0, \tau} v_{0, y y y}+\frac{1}{3} v_{0, \tau} u_{0, y y y},
\end{aligned}
$$

which, on using (5.10), give

$$
\begin{aligned}
& 0=u_{1, \tau}+2 \phi v_{1, \tau}+\theta u_{1, y}-2 \theta \phi v_{1, y}, \\
& 0=\phi u_{1, \tau}+v_{1, \tau}-\theta \phi u_{1, y}+\theta v_{1, y} .
\end{aligned}
$$

If we assume this system of linear equations has travelling wave solutions for both $u_{1}$ and $v_{1}$, that is

$$
u_{1}=u_{1}(z), \quad v_{1}=v_{1}(z), \quad \text { with } \quad z=y-c \tau,
$$

we find

$$
\mathbf{M}\left(\begin{array}{l}
u_{1}^{\prime}(z) \\
v_{1}^{\prime}(z)
\end{array}\right)=\left(\begin{array}{l}
0 \\
0
\end{array}\right), \quad \text { where } \quad \mathbf{M}=\left(\begin{array}{cc}
\theta-c & -2 \theta \phi-2 \phi c \\
-\theta \phi-\phi c & \theta-c
\end{array}\right) .
$$

In order for nontrivial solutions to exist, that is, $\left(u_{1}^{\prime}, v_{1}^{\prime}\right) \neq(0,0)$, we require $\operatorname{det}(\mathbf{M})=0$, which implies

$$
(\theta-c)^{2}=2 \phi^{2}(\theta+c)^{2},
$$

and so the two characteristic speeds $c_{1}, c_{2}$ are given by the 'generalised eigenvalues'

$$
c_{1}=\frac{\theta(1+\phi \sqrt{2})}{(1-\phi \sqrt{2})}=\frac{a}{2 k^{2}(1-\phi \sqrt{2})^{2}}, \quad c_{2}=\frac{\theta(1-\phi \sqrt{2})}{(1+\phi \sqrt{2})}=\frac{a}{2 k^{2}(1+\phi \sqrt{2})^{2}} .
$$

Since $c_{1} c_{2}=\theta^{2}$, the velocities $c_{1}, c_{2}$ have the same sign, and in each of the limits $\phi \rightarrow \pm 1 / \sqrt{2}$, one of the characteristic velocities $c_{1}, c_{2} \rightarrow \pm \infty$. Later calculations are simplified by noting

$$
\begin{gathered}
c_{2}-c_{1}=\frac{-4 \sqrt{2} \phi \theta}{\left(1-2 \phi^{2}\right)}, \quad \theta-c_{1}=\frac{-2 \theta \phi \sqrt{2}}{(1-\phi \sqrt{2})}, \quad \theta-c_{2}=\frac{2 \theta \phi \sqrt{2}}{(1+\phi \sqrt{2})}, \\
\theta+c_{1}=\frac{2 \theta}{(1-\phi \sqrt{2})}, \quad \theta+c_{2}=\frac{2 \theta}{(1+\phi \sqrt{2})} .
\end{gathered}
$$

The degenerate case $c=c_{1}=c_{2}$ corresponds to $\phi=0$ and although the eigenvalue is repeated, there remain two distinct eigenvectors. In this case, the leading order solution vanishes, that is, $v_{0}=0$, from (5.10); however, a nontrivial first correction term remains possible, that is, $v_{1} \neq 0$.

Corresponding to each characteristic speed there is a 'generalised eigenvector'

$$
\mathbf{f}_{1}=\left(\begin{array}{c}
u_{1}^{\prime} \\
v_{1}^{\prime}
\end{array}\right)=\left(\begin{array}{c}
\sqrt{2} \\
-1
\end{array}\right), \quad \text { and } \quad \mathbf{f}_{2}=\left(\begin{array}{c}
u_{1}^{\prime} \\
v_{1}^{\prime}
\end{array}\right)=\left(\begin{array}{c}
\sqrt{2} \\
1
\end{array}\right)
$$


respectively, each satisfying $\mathbf{M f}_{j}=\mathbf{0}$. At this point it is convenient to transform from the independent variables $(y, \tau)$ to the two travelling wave coordinates, one for each of the characteristic velocities (5.19). We define

$$
z=y-c_{1} \tau, \quad w=y-c_{2} \tau, \quad \tau=\frac{z-w}{c_{2}-c_{1}}, \quad y=\frac{c_{2} z-c_{1} w}{c_{2}-c_{1}}
$$

which implies the derivatives transform according to $\partial_{y}=\partial_{z}+\partial_{w}$, and $\partial_{\tau}=-c_{1} \partial_{z}-c_{2} \partial_{w}$.

The dependent variables $u_{1}, v_{1}$ are replaced by new variables $q_{1}(z), q_{2}(w)$ so that our solution of (5.14)-(5.15) is expressed as

$$
\left(\begin{array}{l}
u_{1}(y, \tau) \\
v_{1}(y, \tau)
\end{array}\right)=q_{1}(z)\left(\begin{array}{c}
\sqrt{2} \\
-1
\end{array}\right)+q_{2}(w)\left(\begin{array}{c}
\sqrt{2} \\
1
\end{array}\right) .
$$

In component form, this is written

$$
u_{1}(y, \tau)=\sqrt{2} q_{1}(z)+\sqrt{2} q_{2}(w), \quad v_{1}=-q_{1}(z)+q_{2}(w),
$$

and the transformations in derivatives explicitly as

$$
\begin{array}{ll}
u_{1, \tau}=-\sqrt{2}\left(c_{1} q_{1}^{\prime}(z)+c_{2} q_{2}^{\prime}(w)\right), & v_{1, \tau}=c_{1} q_{1}^{\prime}(z)-c_{2} q_{2}^{\prime}(w), \\
u_{1, y}=\sqrt{2}\left(q_{1}^{\prime}(z)+q_{2}^{\prime}(w)\right), & v_{1, y}=-q_{1}^{\prime}(z)+q_{2}^{\prime}(w) .
\end{array}
$$

Having this form for our solution of (5.14) and (5.15) but with the profiles $q_{1}(\cdot)$ and $q_{2}(\cdot)$ still undetermined, we proceed to the highest order terms considered here in order to find equations which involve higher derivatives to provide the shape of these functions.

\subsection{Third order terms}

At $O\left(h^{-1}\right)$, we obtain the following terms from (5.8):

$$
\begin{array}{rl}
y= & 2 u_{2, \tau} u_{0, y}+2 u_{1, \tau} u_{1, y}+\frac{1}{3} u_{1, \tau} u_{0, y y y}+2 u_{0, \tau} u_{2, y}+\frac{1}{3} u_{0, \tau} u_{1, y y y}+\frac{1}{60} u_{0, \tau} u_{0, y y y y y} \\
& +4 v_{2, \tau} v_{0, y}+4 v_{1, \tau} v_{1, y}+\frac{2}{3} v_{1, \tau} v_{0, y y y}+4 v_{0, \tau} v_{2, y}+\frac{2}{3} v_{0, \tau} v_{1, y y y}+\frac{1}{30} v_{0, \tau} v_{0, y y y y y}, \\
0 & 2 u_{2, \tau} v_{0, y}+2 u_{1, \tau} v_{1, y}+\frac{1}{3} u_{1, \tau} v_{0, y y y}+2 u_{0, \tau} v_{2, y}+\frac{1}{3} u_{0, \tau} v_{1, y y y}+\frac{1}{60} u_{0, \tau} v_{0, y y y y y} \\
& +2 v_{2, \tau} u_{0, y}+2 v_{1, \tau} u_{1, y}+\frac{1}{3} v_{1, \tau} u_{0, y y y}+2 v_{0, \tau} u_{2, y}+\frac{1}{3} v_{0, \tau} u_{1, y y y}+\frac{1}{60} v_{0, \tau} u_{0, y y y y y} .
\end{array}
$$

Since the first derivatives of $u_{0}, v_{0}$ are constants and higher derivatives vanish, using the solution (5.10) simplifies (5.26) to

$$
\begin{aligned}
\frac{y}{2 k} & =u_{2, \tau}+\frac{u_{1, \tau} u_{1, y}}{k}+\theta u_{2, y}+\frac{1}{6} \theta u_{1, y y y}+2 \phi v_{2, \tau}+\frac{2 v_{1, \tau} v_{1, y}}{k}-2 \phi \theta v_{2, y}-\frac{1}{3} \phi \theta v_{1, y y y}, \\
0 & =\phi u_{2, \tau}+\frac{u_{1, \tau} v_{1, y}}{k}+\theta v_{2, y}+\frac{1}{6} \theta v_{1, y y y}+v_{2, \tau}+\frac{v_{1, \tau} u_{1, y}}{k}-\theta \phi u_{2, y}-\frac{1}{6} \theta \phi u_{1, y y y} .
\end{aligned}
$$

Using (5.24) to eliminate $u_{1}, v_{1}$ in favour of $q_{1}(z), q_{2}(w)$, significantly simplifies (5.27) to

$$
\begin{aligned}
\frac{c_{2} z-c_{1} w}{2 k\left(c_{2}-c_{1}\right)}= & \frac{1}{6} \theta \sqrt{2}\left[(1+\phi \sqrt{2}) q_{1}^{\prime \prime \prime}(z)+(1-\sqrt{2} \phi) q_{2}^{\prime \prime \prime}(w)\right]-\frac{4}{k}\left(c_{1} q_{1}^{\prime}(z)^{2}+c_{2} q_{2}^{\prime}(w)^{2}\right) \\
& +\left(\theta-c_{1}\right) u_{2, z}+\left(\theta-c_{2}\right) u_{2, w}-2 \phi\left(\theta+c_{1}\right) v_{2, z}-2 \phi\left(\theta+c_{2}\right) v_{2, w} \\
0= & \frac{1}{6} \theta\left[(1-\sqrt{2} \phi) q_{2}^{\prime \prime \prime}(w)-(1+\sqrt{2} \phi) q_{1}^{\prime \prime \prime}(z)\right]+\frac{2 \sqrt{2}}{k}\left(c_{1} q_{1}^{\prime}(z)^{2}-c_{2} q_{2}^{\prime}(w)^{2}\right) \\
& -\phi\left(\theta+c_{1}\right) u_{2, z}-\phi\left(\theta+c_{2}\right) u_{2, w}+\left(\theta-c_{1}\right) v_{2, z}+\left(\theta-c_{2}\right) v_{2, w} .
\end{aligned}
$$


These equations retain partial derivatives of the higher-order correction terms $u_{2}, v_{2}$, which are also now considered as functions of $z, w$.

We treat (5.28) as a pair of coupled third-order ordinary differential equations for $q_{1}(z)$ and $q_{2}(w)$, writing them as

$$
\begin{gathered}
\frac{1}{6} \theta \mathbf{M}\left(\begin{array}{c}
q_{1}^{\prime \prime \prime}(z) \\
q_{2}^{\prime \prime \prime}(w)
\end{array}\right)=\mathbf{r}, \quad \text { where } \quad \mathbf{M}=\left(\begin{array}{cc}
\sqrt{2}(1+\phi \sqrt{2}) & \sqrt{2}(1-\phi \sqrt{2}) \\
-(1+\phi \sqrt{2}) & 1-\phi \sqrt{2}
\end{array}\right), \quad \text { and } \\
\mathbf{r}=\left(\begin{array}{c}
\frac{\left(c_{2} z-c_{1} w\right)}{2 k\left(c_{2}-c_{1}\right)}+\frac{4}{k}\left(c_{1} q_{1}^{\prime 2}+c_{2} q_{2}^{\prime 2}\right)-u_{2, z}\left(\theta-c_{1}\right)-u_{2, w}\left(\theta-c_{2}\right)+2 \phi v_{2, z}\left(\theta+c_{1}\right)+2 \phi v_{2, w}\left(\theta+c_{2}\right) \\
-\frac{2 \sqrt{2}}{k}\left(c_{1} q_{1}^{\prime 2}-c_{2} q_{2}^{\prime 2}\right)-v_{2, z}\left(\theta-c_{1}\right)-v_{2, w}\left(\theta-c_{2}\right)+\phi u_{2, z}\left(\theta+c_{1}\right)+\phi u_{2, w}\left(\theta+c_{2}\right)
\end{array}\right) .
\end{gathered}
$$

Inverting the matrix $\mathbf{M}$ and calculating $\mathbf{M}^{-1} \mathbf{r}$, from (5.29)-(5.30), using (5.20) we obtain

$$
\begin{aligned}
\frac{1}{3} \theta \sqrt{2}\left(1-2 \phi^{2}\right)\left(\begin{array}{l}
q_{1}^{\prime \prime \prime}(z) \\
q_{2}^{\prime \prime \prime}(w)
\end{array}\right)= & \frac{\left(1-2 \phi^{2}\right)\left(c_{1} w-c_{2} z\right)}{8 \sqrt{2} k \phi \theta}\left(\begin{array}{l}
(1-\phi \sqrt{2}) \\
(1+\phi \sqrt{2})
\end{array}\right)+\frac{8}{k}\left(\begin{array}{l}
c_{1} q_{1}^{\prime}(z)^{2}(1-\phi \sqrt{2}) \\
c_{2} q_{2}^{\prime}(w)^{2}(1+\phi \sqrt{2})
\end{array}\right) \\
& +\frac{4 \sqrt{2} \theta \phi}{\left(1-2 \phi^{2}\right)}\left(\begin{array}{c}
\left(\sqrt{2} v_{2, w}-u_{2, w}\right)(1-\phi \sqrt{2})^{2} \\
\left(\sqrt{2} v_{2, z}+u_{2, z}\right)(1+\phi \sqrt{2})^{2}
\end{array}\right) .
\end{aligned}
$$

Note that the expression for $q_{1}^{\prime \prime \prime}(z)$ only involves a quadratic term $q_{1}^{\prime}(z)^{2}$, with no dependence on $q_{2}^{\prime}(w)$; similarly, the expression for $q_{2}^{\prime \prime \prime}(w)$ only involves a quadratic term $q_{2}^{\prime}(w)^{2}$, with no dependence on $q_{1}^{\prime}(z)$. However, the equation in $q_{1}(z)$ has terms which apparently depend also on $w$, and the equation in $q_{2}(w)$ has terms apparently dependent also on $z$ : as we now see, this cannot in fact be the case. Let us write these equations as

$$
\frac{1}{3} \theta \sqrt{2}\left(1-2 \phi^{2}\right)\left(\begin{array}{c}
q_{1}^{\prime \prime \prime}(z) \\
q_{2}^{\prime \prime \prime}(w)
\end{array}\right)=\left(\begin{array}{l}
A \\
\widetilde{A}
\end{array}\right)\left(c_{1} w-c_{2} z\right)+\frac{8}{k}\left(\begin{array}{c}
c_{1} q_{1}^{\prime}(z)^{2}(1-\phi \sqrt{2}) \\
c_{2} q_{2}^{\prime}(w)^{2}(1+\phi \sqrt{2})
\end{array}\right)+\left(\begin{array}{c}
B\left(\sqrt{2} v_{2, w}-u_{2, w}\right) \\
\widetilde{B}\left(\sqrt{2} v_{2, z}+u_{2, z}\right)
\end{array}\right)
$$

where

$$
\begin{aligned}
A=\frac{\left(1-2 \phi^{2}\right)(1-\phi \sqrt{2})}{8 \sqrt{2} k \phi \theta}, & \widetilde{A}=\frac{\left(1-2 \phi^{2}\right)(1+\phi \sqrt{2})}{8 \sqrt{2} k \phi \theta}, \\
B=\frac{4 \sqrt{2} \theta \phi(1-\phi \sqrt{2})^{2}}{\left(1-2 \phi^{2}\right)}, & \widetilde{B}=\frac{4 \sqrt{2} \theta \phi(1+\phi \sqrt{2})^{2}}{\left(1-2 \phi^{2}\right)} .
\end{aligned}
$$

Differentiating the first component equation of (5.32) with respect to $w$ yields

$$
\sqrt{2} v_{2, w w}-u_{2, w w}=-\frac{c_{1} A}{B}
$$

and differentiating the second component equation of (5.32) with respect to $z$ yields

$$
\sqrt{2} v_{2, z z}+u_{2, z z}=\frac{c_{2} \widetilde{A}}{\widetilde{B}},
$$


and thus we obtain

$$
\begin{aligned}
& \sqrt{2} v_{2}-u_{2}=-\frac{c_{1} A}{2 B} w^{2}+d_{1}(z) w+d_{0}(z), \\
& \sqrt{2} v_{2}+u_{2}=\frac{c_{2} \widetilde{A}}{2 \widetilde{B}} z^{2}+\widetilde{d}_{1}(w) z+\widetilde{d}_{0}(w) .
\end{aligned}
$$

Elimination between these equations yields $u_{2}$ and $v_{2}$, although it is the above combinations that are of relevance here. Substituting into (5.32) then gives the following system, the first equation of which does not in fact depend on $w$, and the second equation of which does not in fact depend on $z$ :

$$
\frac{1}{3} \theta \sqrt{2}\left(1-2 \phi^{2}\right)\left(\begin{array}{c}
q_{1}^{\prime \prime \prime}(z) \\
q_{2}^{\prime \prime \prime}(w)
\end{array}\right)=\frac{8}{k}\left(\begin{array}{c}
c_{1} q_{1}^{\prime}(z)^{2}(1-\phi \sqrt{2}) \\
c_{2} q_{2}^{\prime}(w)^{2}(1+\phi \sqrt{2})
\end{array}\right)+\left(\begin{array}{c}
B d_{1}(z)-c_{2} A z \\
\widetilde{B} \widetilde{d}_{1}(w)+c_{1} \widetilde{A} w
\end{array}\right) .
$$

Choosing $d_{1}(z)$ and $\widetilde{d}_{1}(w)$ to be linear in $z$ and $w$ respectively then gives rise to a pair of uncoupled first Painlevé equations, but in the distinct independent variables $z$ and $w$.

\section{Solution classes of matrix $\mathbf{P}_{I I}$}

It is well-known that the scalar $P_{I I}$ equation has rational solutions for integer values of the parameter therein, as well as solutions expressible in terms of Airy functions for half-oddinteger values of this parameter. This then leads naturally to the question of the classes of solutions which can be obtained for matrix $P_{I I}$ (we note that the scalar $P_{I}$ equation does not have any solutions expressible in terms of classical functions, and so we do not consider matrix $P_{I}$ here). As in the case of scalar $P_{I I}$, we seek to provide an answer to this question by obtaining initial solutions of matrix $P_{I I}$ and using aBTs to generate further solutions. We begin with a general discussion of the aBTs of matrix $P_{I I}$, and then discuss possible initial solutions and their iteration using aBTs. We remark that no results on solution classes of matrix $P_{I I}$ have previously been published.

\subsection{Auto-Bäcklund transformations}

Equation (1.1) has the following aBTs:

$$
\begin{array}{ll}
f: \quad \mathbf{u}=\mathbf{v}+\frac{1}{2}(\alpha-\widetilde{\alpha})\left(\mathbf{v}_{x}-\mathbf{v}^{2}+\frac{1}{2} c_{0} \mathbf{I}+\mathbf{F}_{0}+g_{0} \mathbf{I} x\right)^{-1}, \quad \alpha=-\widetilde{\alpha}-2 g_{0}, \quad \mathbf{E}_{0}=\mathbf{F}_{0}, \\
g: \quad \mathbf{u}=-\mathbf{v}, \quad \alpha=-\widetilde{\alpha}, \quad \mathbf{E}_{0}=\mathbf{F}_{0}, \\
k: \quad \mathbf{u}=\mathbf{v}^{T}, \quad \alpha=\widetilde{\alpha}, \quad \mathbf{E}_{0}=\mathbf{F}_{0}^{T},
\end{array}
$$

which map from equation (1.1) in $\left(\mathbf{v}, \widetilde{\alpha}, \mathbf{F}_{0}\right)$ to the same equation in $\left(\mathbf{u}, \alpha, \mathbf{E}_{0}\right)$. The aBTs $f(6.1)$ and $g$ (6.2) correspond to the aBTs $f$ and $g$ given in [1] for the matrix hierarchy of which (1.1) is the first member. The aBT $k$ (6.3) corresponds to a generalization of the transformation $h-$ for which $\mathbf{F}_{0}$ was assumed to be symmetric — given in [1] for this matrix hierarchy. The above 
transformation $k$ in fact also holds for this matrix hierarchy and not just for equation (1.1). ${ }^{1}$ (The aBTs (6.1) - (6.3) correspond similarly to the aBTs $F$ and $G$ and a generalization of the aBT $H$, for which the matrix coefficent $E$ was assumed to be symmetric, given for a matrix PDE in [6].)

The group of aBTs (of (1.1) and its hierarchy) generated by $f, g$ and $k$ has the presentation

$$
G=\left\langle f, g, k ; f^{2}=g^{2}=k^{2}=(f k)^{2}=(g k)^{2}=1\right\rangle,
$$

and is isomorphic to the direct product of the affine Weyl group of type $A_{1}^{(1)}$ with the cyclic group $\mathbb{Z}_{2}$, i.e., $G \cong A_{1}^{(1)} \times Z_{2}$. We now define the transformations $r=g f$ and $s=f g$. It follows from the relations in (6.4) that any composition of $f, g$ and $k$ can be written either as

$$
k^{\epsilon_{1}} f^{\epsilon_{2}} r^{n} \quad \text { or } \quad k^{\epsilon_{1}} g^{\epsilon_{2}} s^{n}, \quad \text { where in either case } \epsilon_{1}, \epsilon_{2} \in\{0,1\}, n \in\{0,1,2, \ldots\} .
$$

This will prove useful later when we dicuss the iteration of solutions using aBTs.

Here we are interested in exact solutions of the matrix analogue of the second Painlevé equation and so take $g_{0}=-1 / 2$ (the autonomous case $g_{0}=0$ of (1.1) will be discussed elsewhere). In order to simplify the discussion in the following subsection, we also make the change of variable $x=z+c_{0}$ and so will be dealing with the ODE

$$
\mathbf{u}_{z z}-2 \mathbf{u}^{3}+\mathbf{u} \mathbf{E}_{0}+\mathbf{E}_{0} \mathbf{u}-z \mathbf{u}-\alpha \mathbf{I}=\mathbf{0}
$$

for which we have the aBTs $g$ (6.2) and $k$ (6.3) as well as $f, r$ and $s$, these last three now being written

$$
\begin{array}{ccc}
f: & \mathbf{u}=\mathbf{v}+\frac{1}{2}(\alpha-\widetilde{\alpha})\left(\mathbf{v}_{z}-\mathbf{v}^{2}+\mathbf{F}_{0}-\frac{1}{2} \mathbf{I} z\right)^{-1}, \quad \alpha=-\widetilde{\alpha}+1, & \mathbf{E}_{0}=\mathbf{F}_{0}, \\
r=g f: & \mathbf{u}=-\mathbf{v}+\frac{1}{2}(\alpha+\widetilde{\alpha})\left(\mathbf{v}_{z}-\mathbf{v}^{2}+\mathbf{F}_{0}-\frac{1}{2} \mathbf{I} z\right)^{-1}, \quad \alpha=\widetilde{\alpha}-1, & \mathbf{E}_{0}=\mathbf{F}_{0}, \\
s=f g: & \mathbf{u}=-\mathbf{v}+\frac{1}{2}(\alpha+\widetilde{\alpha})\left(-\mathbf{v}_{z}-\mathbf{v}^{2}+\mathbf{F}_{0}-\frac{1}{2} \mathbf{I} z\right)^{-1}, \quad \alpha=\widetilde{\alpha}+1, \quad \mathbf{E}_{0}=\mathbf{F}_{0},
\end{array}
$$

The last two of these aBTs (identifying $x=z+c_{0}$, and noting that in both $\mathbf{E}_{0}=\mathbf{F}_{0}$ ) correspond respectively to the aBTs (1.6)-(1.8) and (1.9)—(1.11) for matrix $P_{I I}$ in the form (1.5).

\subsection{On the iteration of initial solutions}

Let us now consider the question of obtaining initial and iterated solutions of the matrix $P_{I I}$ ODE

$$
\widehat{\mathbf{v}}_{z z}-2 \widehat{\mathbf{v}}^{3}+\widehat{\mathbf{v}}_{0}+\widehat{\mathbf{F}}_{0} \widehat{\mathbf{v}}-z \widehat{\mathbf{v}}-\widehat{\beta} \mathbf{I}=\mathbf{0},
$$

where $\widehat{\mathbf{F}}_{0}$ is an arbitrary (in general complex) constant matrix. As a first step we note that, since the transformation $p$ given by

$$
p: \quad \widehat{\mathbf{v}}=\mathbf{P v P}^{-1}, \quad \widehat{\beta}=\beta, \quad \widehat{\mathbf{F}}_{0}=\mathbf{P F}_{0} \mathbf{P}^{-1},
$$

\footnotetext{
${ }^{1}$ In general $k$ changes $\mathbf{F}_{0}$, answering the question which arose in an intervention in A. Pickering's talk at Group Analysis of Differential Equations and Integrable Systems (Cyprus, 2018) of whether such an aBT might exist.
} 
where $\mathbf{P}$ is a nonsingular constant matrix, maps from

$$
\mathbf{v}_{z z}-2 \mathbf{v}^{3}+\mathbf{v} \mathbf{F}_{0}+\mathbf{F}_{0} \mathbf{v}-z \mathbf{v}-\beta \mathbf{I}=\mathbf{0}
$$

to (6.10), we can always transform to an equation (6.12) with the matrix $\mathbf{F}_{0}$ being similar to $\widehat{\mathbf{F}}_{0}$. The most obvious choice is to take $\mathbf{F}_{0}$ in Jordan canonical form. In particular, if $\widehat{\mathbf{F}}_{0}$ is a normal matrix (i.e., $\widehat{\mathbf{F}}_{0}^{*} \widehat{\mathbf{F}}_{0}=\widehat{\mathbf{F}}_{0} \widehat{\mathbf{F}}_{0}^{*}$, where $\widehat{\mathbf{F}}_{0}^{*}$ is the conjugate transpose of $\widehat{\mathbf{F}}_{0}$ ), then we may take $\mathbf{F}_{0}$ to be diagonal. Normal matrices include Hermitian and real symmetric matrices (where for such $\widehat{\mathbf{F}}_{0}$ the diagonal matrix $\mathbf{F}_{0}$ is real), and skew-Hermitian and real skew-symmetric matrices (where for such $\widehat{\mathbf{F}}_{0}$ the diagonal matrix $\mathbf{F}_{0}$ is pure imaginary). However, $\mathbf{F}_{0}$ may in fact be taken to be any matrix similar to $\widehat{\mathbf{F}}_{0}$, e.g., we may always assume $\mathbf{F}_{0}$ to be upper-triangular, or symmetric. (Rather than think of $p$ as an aBT which changes $\widehat{\mathbf{F}}_{0}$, we prefer to regard it as allowing a form for $\widehat{\mathbf{F}}_{0}$ to be chosen; this form may be canonical or otherwise, essentially unique or otherwise.)

Let us now observe that the transformation $p$ (6.11) commutes with the aBTs $g$ (6.2) and $f$ (6.7), and so also with the aBTs $r=g f$ (6.8) and $s=f g$ (6.9). This means that the effect of acting with any composition of these four aBTs $g, f, r$ and $s$ can be calculated equally at the level of equation (6.10) or at the level of equation (6.12). However, the transformation $p$ does not commute with the aBT $k$ (6.3) unless $\mathbf{P}^{T} \mathbf{P}$ commutes with $\mathbf{F}_{0}$ and $\mathbf{v}$, i.e., unless $\mathbf{P}^{T} \mathbf{P}=\gamma \mathbf{I} \neq \mathbf{0}$.

We may proceed as follows. Beginning with equation (6.10) we use the transformation $p^{-1}$ to obtain an equivalent equation (6.12) with $\mathbf{F}_{0}$ similar to $\widehat{\mathbf{F}}_{0}$. We may use an ansatz for the form of $\mathbf{v}$ to obtain a solution $\mathbf{v}_{0}$ of (6.12). If we take $\mathbf{F}_{0}$ to be in Jordan canonical form or simply upper-triangular, then we may use as an ansatz that $\mathbf{v}_{0}$ is upper-triangular. If we take $\mathbf{F}_{0}$ to be symmetric, then we may use as an ansatz that $\mathbf{v}_{0}$ is symmetric (the result of substituting symmetric $\mathbf{F}_{0}$ and $\mathbf{v}_{0}$ into (6.12) is also symmetric). Given $\mathbf{v}_{0}$, we then have a solution $\widehat{\mathbf{v}}_{0}=p \mathbf{v}_{0}$ of (6.10). However, instead of calculating the effect of one of the compositions of aBTs (6.5) on $\widehat{\mathbf{v}}_{0}$ we iterate at the level of equation (6.12) and return to (6.10) via $p$, since we have that

$$
k^{\epsilon_{1}} f^{\epsilon_{2}} r^{n} \widehat{\mathbf{v}}_{0}=k^{\epsilon_{1}} p f^{\epsilon_{2}} r^{n} \mathbf{v}_{0} \quad \text { and } \quad k^{\epsilon_{1}} g^{\epsilon_{2}} s^{n} \widehat{\mathbf{v}}_{0}=k^{\epsilon_{1}} p g^{\epsilon_{2}} s^{n} \mathbf{v}_{0} .
$$

In each case the action of $k$ (if $\epsilon_{1}=1$ ) is calculated as the final step at the level of the original equation (6.10). Using the procedure outlined here we expect to simplify the expressions obtained when iterating solutions. Except possibly for $k$ as the final step (if $\epsilon_{1}=1$ ), the actions of the compositions of aBTs in (6.13) on $\mathbf{v}_{0}$ only involve $\mathbf{F}_{0}$ (in general expected to be simpler than $\widehat{\mathbf{F}}_{0}$ ).

We note in passing that the same observations as made here for the matrix $P_{I I}$ equation also hold for the matrix $P_{I I}$ hierarchy given in [1]: with $g_{0}=-1 / 2$ we can shift $x$ to remove $c_{0}$ and then use the transformation $p^{-1}$ to obtain an equation involving a matrix similar to the constant matrix $E$ in our matrix $P_{I I}$ hierarchy, for which we obtain (e.g., via an ansatz) a solution $\mathbf{v}_{0}$; since $p$ commutes with the aBTs $f$ and $g$ given in [1] we then calculate the effect of compositions of $f, g$ and $k$ on a solution $\widehat{\mathbf{v}}_{0}=p \mathbf{v}_{0}$ of the original equation by iterating at the level of the transformed equation and returning to the original equation via $p$ as in (6.13). We will consider this extension to the study of classes of solutions of the matrix $P_{I I}$ hierarchy in a later paper. 


\subsection{Classes of initial solutions}

Let us now consider the problem of finding initial solutions $\mathbf{v}_{0}$ of equation (6.12), $\mathbf{F}_{0}$ being similar to some original $\widehat{\mathbf{F}}_{0}$. There are two obvious classes of initial solutions that we may consider:

for parameter value $\beta=0, \quad \mathbf{v}_{0}=\mathbf{0}$;

for parameter value $\beta=\frac{1}{2}, \quad \mathbf{v}_{0}$ general solution of $\mathbf{v}_{z}-\mathbf{v}^{2}+\mathbf{F}_{0}-\frac{1}{2} z \mathbf{I}=\mathbf{0}$.

(Instead of the basic special integral in (6.15), we could take $\mathbf{v}_{0}$ to be the general solution of $-\mathbf{v}_{z}-\mathbf{v}^{2}+\mathbf{F}_{0}-\frac{1}{2} z \mathbf{I}=\mathbf{0}$ for parameter value $\beta=-\frac{1}{2}$. However, (6.15) will serve our purposes here.) Corresponding to (6.14) and (6.15) we have solutions $\widehat{\mathbf{v}}_{0}=p \mathbf{v}_{0}$ of (6.10), i.e., $\widehat{\mathbf{v}}_{0}=$ $p \mathbf{v}_{0}=\mathbf{0}$ for $\widehat{\beta}=0$, and $\widehat{\mathbf{v}}_{0}=p \mathbf{v}_{0}$ the corresponding solution of $\widehat{\mathbf{v}}_{z}-\widehat{\mathbf{v}}^{2}+\widehat{\mathbf{F}}_{0}-\frac{1}{2} z \mathbf{I}=\mathbf{0}$ for $\widehat{\beta}=\frac{1}{2}$.

Let us consider the first case (6.14). We assume without loss of generality that in equation (6.12) $\mathbf{F}_{0}$ has been taken to be upper triangular or in Jordan canonical form. Since $\mathbf{v}_{0}, \mathbf{F}_{0}$ and $\mathbf{I} z$ are upper triangular, then so are the solutions $f^{\epsilon_{2}} r^{n} \mathbf{v}_{0}$ and $g^{\epsilon_{2}} s^{n} \mathbf{v}_{0}$ of (6.12) given in (6.13). The diagonal elements of these solutions are known rational solutions of scalar second Painlevé equations,

$$
v_{i i, z z}-2 v_{i i}^{3}-\left(z-2 f_{i i}\right) v_{i i}-\beta=0,
$$

as given by the diagonal elements of (6.12) for upper triangular matrices $\mathbf{v}=\left(v_{i j}\right)$ and $\mathbf{F}_{0}=$ $\left(f_{i j}\right)$. These rational solutions are the result of corresponding iterations, with initial solutions $v_{i i}=0$ of $(6.16)$ for $\beta=0$, of the aBTs

$$
u_{i i}=-v_{i i}, \quad \alpha=-\widetilde{\alpha},
$$

and

$$
\left\{\begin{array} { l } 
{ u _ { i i } = v _ { i i } + \frac { 1 } { 2 } ( \alpha - \widetilde { \alpha } ) G _ { i i } ^ { - 1 } , } \\
{ \alpha = - \widetilde { \alpha } + 1 , }
\end{array} \quad \left\{\begin{array} { l } 
{ u _ { i i } = - v _ { i i } + \frac { 1 } { 2 } ( \alpha + \widetilde { \alpha } ) G _ { i i } ^ { - 1 } , } \\
{ \alpha = \widetilde { \alpha } - 1 , }
\end{array} \quad \left\{\begin{array}{l}
u_{i i}=-v_{i i}+\frac{1}{2}(\alpha+\widetilde{\alpha}) H_{i i}^{-1}, \\
\alpha=\widetilde{\alpha}+1,
\end{array}\right.\right.\right.
$$

these aBTs being obtained as the diagonal elements of the aBTs (6.2) and (6.7) -(6.9) where we have set

$$
\mathbf{G}=\mathbf{v}_{z}-\mathbf{v}^{2}+\mathbf{F}_{0}-\frac{1}{2} \mathbf{I} z \text { and } \quad \mathbf{H}=-\mathbf{v}_{z}-\mathbf{v}^{2}+\mathbf{F}_{0}-\frac{1}{2} \mathbf{I} z
$$

Since in the iteration of rational solutions for scalar second Painlevé equations (6.16) we always have that $G_{i i} \neq 0$ and $H_{i i} \neq 0$, it follows that $\operatorname{det} \mathbf{G}=\prod_{i=1}^{m} G_{i i} \neq 0$ and $\operatorname{det} \mathbf{H}=\prod_{i=1}^{m} H_{i i} \neq$ 0 . Thus the inverse matrices in the aBTs (6.7) - (6.9) always exist and we can iterate to obtain solutions $f^{\epsilon_{2}} r^{n} \mathbf{v}_{0}$ and $g^{\epsilon_{2}} s^{n} \mathbf{v}_{0}$ of (6.12). The solutions $r^{n} \mathbf{v}_{0}$ are solutions for parameter values $\beta=-n$, and $s^{n} \mathbf{v}_{0}$ for parameter values $\beta=n$; the solutions $f r^{n-1} \mathbf{v}_{0}$ and $s^{n} \mathbf{v}_{0}$ for parameter values $\beta=n$ are equal, as are the solutions $r^{n} \mathbf{v}_{0}$ and $g s^{n} \mathbf{v}_{0}$ for parameter values $\beta=-n$. In this way we obtain using the aBTs $f$ and $g$ exactly one solution of (6.12) for each integer value of $\beta$. We map back to solutions of (6.10) using the transformation (6.11), to which solutions we can then apply the aBT $k$ if we so wish. The solutions of (6.10) thus obtained are matrix analogues of the well-known known rational solutions of scalar $P_{I I}$ for integer values of its parameter. 
Let us now consider the second case (6.15). Again we assume without loss of generality that $\mathbf{F}_{0}$ in equation (6.12) has been taken to be upper triangular or in Jordan canonical form. We iterate using the aBT $s$ (6.9) in order to obtain solutions $s^{n} \mathbf{v}_{0}$ of (6.12) for parameter values $\beta=n+\frac{1}{2}$, where $\mathbf{v}_{0}$ is the general solution of the basic special integral in (6.15) obtained by linearizing this last equation via $\mathbf{v}=-\mathbf{y}_{z} \mathbf{y}^{-1}$ onto the matrix Airy equation

$$
\mathbf{y}_{z z}=\left(\mathbf{F}_{0}-\frac{1}{2} \mathbf{I} z\right) \mathbf{y}
$$

We note that at each step of this iteration using the aBT $s$ the matrix $\mathbf{H}$ must be nonsingular, since that is the case when $\mathbf{v}_{0}$, instead of being chosen to be the general solution of the basic special integral in (6.15), is chosen to be the particular solution obtained from the solution $\mathbf{y}$ of the above matrix Airy equation having all entries below the main diagonal equal to zero, as we now explain. In this particular case, since $\mathbf{v}_{0}, \mathbf{F}_{0}$ and $\mathbf{I} z$ are upper triangular, so are all solutions $s^{n} \mathbf{v}_{0}$ of (6.12) given in (6.13): the diagonal elements of these solutions give known solutions of scalar second Painlevé equations (6.16) expressible in terms of Airy functions. These solutions of (6.16) are the result of corresponding iterations of the third aBT in (6.18), with initial solutions of the scalar second Painlevé equations (6.16) obtained for $\beta=\frac{1}{2}$ as solutions of $v_{i i, z}-v_{i i}^{2}+f_{i i}-\frac{1}{2} z=0$, and so as $v_{i i}=-y_{i i, z} y_{i i}^{-1}$ where $y_{i i}$ is the general solution of $y_{i i, z z}=\left(f_{i i}-\frac{1}{2} z\right) y_{i i}$. Since in the iteration of such solutions for scalar second Painlevé equations (6.16) we always have $H_{i i} \neq 0$, it follows that $\operatorname{det} \mathbf{H}=\prod_{i=1}^{m} H_{i i} \neq 0$. Thus, since for this particular solution $\mathbf{v}_{0}$ the inverse matrix in the aBT (6.9) always exists, this must also be the case when $\mathbf{v}_{0}$ is the general solution of the basic special integral in (6.15) and there is therefore no obstacle to iterating from such a general solution $\mathbf{v}_{0}$ to obtain solutions $s^{n} \mathbf{v}_{0}$ of (6.12) for parameter values $\beta=n+\frac{1}{2}$. The aBT $g$ then gives solutions $g s^{n} \mathbf{v}_{0}$ of (6.12) for parameter values $\beta=-n-\frac{1}{2}$. We note that we could also, if we define $f \mathbf{v}_{0}=\mathbf{v}_{0}$ (note that if $\mathbf{v}_{0}$ satisfies (6.15) then the matrix whose inverse appears in $f$ is the zero matrix, but since the new parameter value obtained using $f$ is also $\beta=\frac{1}{2}$ then the difference in parameters appearing in the expression for the new solution in $f$ is zero), iterate using $r$ to obtain solutions $r^{n+1} \mathbf{v}_{0}$ for parameter values $\beta=-n-\frac{1}{2}$. The solutions $r^{n+1} \mathbf{v}_{0}$ and $g s^{n} \mathbf{v}_{0}$ of (6.12) for parameter values $\beta=-n-\frac{1}{2}$ are equal, as are the solutions $f r^{n} \mathbf{v}_{0}$ and $s^{n} \mathbf{v}_{0}$ for parameter values $\beta=n+\frac{1}{2}$. In this way we obtain using the aBTs $f$ and $g$ exactly one solution of (6.12) for each half-odd-integer value of $\beta$. We then map back to solutions of (6.10) using the transformation (6.11), to which solutions we can then apply the aBT $k$ if we so wish. The solutions of (6.10) thus obtained are matrix analogues of the solutions of the scalar second Painlevé equation, expressible in terms of Airy functions, for half-odd-integer values of its parameter.

Let us now consider two further classes of initial solutions $\mathbf{v}_{0}$ of equation (6.12). Again we assume without loss of generality that $\mathbf{F}_{0}=\left(f_{i j}\right)$ in equation (6.12) has been taken to be upper triangular or in Jordan canonical form. We consider here initial solutions $\mathbf{v}_{0}$ which generalize cases considered above. Let us seek an initial solution $\mathbf{v}_{0}=\left(v_{i j}\right)$ that is upper triangular. Each element $v_{i i}$ of the leading diagonal of $\mathbf{v}_{0}$ then satisfies a scalar second Painlevé equation (6.16), and each element above the leading diagonal of $\mathbf{v}_{0}$, i.e., $v_{i j}$ with $j>i$, satisfies a linear 
differential equation,

$$
v_{i j, z z}-2 \sum_{k=i}^{l} \sum_{l=k}^{j} v_{i k} v_{k l} v_{l j}+\sum_{k=i}^{j} v_{i k} f_{k j}+\sum_{k=i}^{j} f_{i k} v_{k j}-z v_{i j}=0 .
$$

We may therefore seek an upper triangular initial solution $\mathbf{v}_{0}$ having as diagonal elements solutions of scalar second Painlevé equations (6.16) - taking into account that all of these scalar $P_{I I}$ equations have the same parameter $\beta$ - and elements above the leading diagonal solutions of the linear equations (6.21). These linear equations can be recursively solved for the elements of $\mathbf{v}_{0}$ which lie along successive diagonals parallel to the leading diagonal, the last linear equation to be solved being for the upper right-hand-corner element of $\mathbf{v}_{0}$. They are in general non-homogeneous: the homogeneous part of $(6.21)$ is

$$
v_{i j, z z}-2\left(v_{i i}^{2}+v_{i i} v_{j j}+v_{j j}^{2}\right) v_{i j}+\left(f_{i i}+f_{j j}-z\right) v_{i j}=0 .
$$

As initial solutions of (6.16) for the diagonal elements $v_{i i}$ of $\mathbf{v}_{0}$ we may take:

$$
\begin{array}{lll}
\text { for parameter value } & \beta=0, & v_{i i}=0 \\
\text { for parameter value } & \beta=\frac{1}{2}, & v_{i i} \text { general solution of } v_{i i, z}-v_{i i}^{2}+f_{i i}-\frac{1}{2} z=0 .
\end{array}
$$

In the first case (6.23), the homogeneous part of (6.21), i.e., (6.22), reduces to

$$
v_{i j, z z}+\left(f_{i i}+f_{j j}-z\right) v_{i j}=0,
$$

and so the linear equations (6.21) are Airy equations. These Airy equations for $v_{i, i+k}$ are homogeneous for $k=1$, may be homogeneous for $k=2$ (depending on $\mathbf{F}_{0}$ ), and are nonhomogeneous for $k>2$ (due to contributions from the cubic term in (6.21)). Using the same reasoning as used previously in our discussion of the case (6.14), we see that the inverse matrices in the aBTs (6.7)-(6.9) always exist and we can iterate to obtain solutions $f^{\epsilon_{2}} r^{n} \mathbf{v}_{0}$ and $g^{\epsilon_{2}} s^{n} \mathbf{v}_{0}$ of (6.12). The solutions $r^{n} \mathbf{v}_{0}$ are solutions for parameter values $\beta=-n$, and the solutions $s^{n} \mathbf{v}_{0}$ are solutions for parameter values $\beta=n$. The diagonal elements of these solutions are the well-known rational solutions of scalar second Painlevé equations (6.16). However, for this case (6.23), the solutions $f r^{n-1} \mathbf{v}_{0}$ and $s^{n} \mathbf{v}_{0}$ for parameter values $\beta=n$ are different (except in the special case $\mathbf{v}_{0}=\mathbf{0}$, i.e., (6.14)). Similarly, for this case (6.23), the solutions $r^{n} \mathbf{v}_{0}$ and $g s^{n} \mathbf{v}_{0}$ for parameter values $\beta=-n$ are different (except in the special case $\mathbf{v}_{0}=\mathbf{0}$, i.e., (6.14)). In this way we obtain using the aBTs $f$ and $g$ exactly two solutions of (6.12) for each integer value of $\beta$. We then map back to solutions of (6.10) using the transformation (6.11), to which solutions we can then apply the aBT $k$ if we so wish. The solutions of (6.10) thus obtained are generalizations of the matrix analogues of the rational solutions of the scalar $P_{I I}$ equation obtained above using the initial solution (6.14).

In the second case (6.24), the homogeneous part of the linear equation (6.21), i.e., (6.22), is

$$
v_{i j, z z}-2\left(y_{i i, z}^{2} y_{i i}^{-2}+y_{i i, z} y_{i i}^{-1} y_{j j, z} y_{j j}^{-1}+y_{j j, z}^{2} y_{j j}^{-2}\right) v_{i j}+\left(f_{i i}+f_{j j}-z\right) v_{i j}=0 \text {, }
$$


where each $y_{k k}$ is obtained as the general solution of $y_{k k, z z}=\left(f_{k k}-\frac{1}{2} z\right) y_{k k}$ (the linearization of $v_{k k, z}-v_{k k}^{2}+f_{k k}-\frac{1}{2} z=0$ under $\left.v_{k k}=-y_{k k, z} y_{k k}^{-1}\right)$. The linear equations (6.21) for $v_{i, i+k}$ may be homogeneous for $k=1$ (depending on $\mathbf{F}_{0}$ ), and are non-homogeneous for $k>1$ (due to contributions from the cubic term in (6.21)). Using the same reasoning as used previously in our discussion of (the upper triangular subcase of) the initial solution (6.15), we see that the inverse matrices required for repeated application of the aBT (6.9) always exist and we can iterate to obtain solutions $s^{n} \mathbf{v}_{0}$ of (6.12) for parameter values $\beta=n+\frac{1}{2}$. The diagonal elements of these solutions are well-known solutions of scalar second Painlevé equations (6.16) expressible in terms of Airy fuctions. The aBT $g$ then gives solutions $g s^{n} \mathbf{v}_{0}$ of (6.12) for parameter values $\beta=-n-\frac{1}{2}$. As previously, if we define $f \mathbf{v}_{0}=\mathbf{v}_{0}\left(\mathbf{v}_{0}\right.$ is such that the matrix whose inverse appears in $f$ has zero determinant, but since the new parameter value obtained using $f$ is also $\beta=\frac{1}{2}$ then the difference in parameters appearing in the expression for the new solution in $f$ is zero), we may iterate using $r$ to obtain solutions $r^{n+1} \mathbf{v}_{0}=g s^{n} \mathbf{v}_{0}$ for parameter values $\beta=-n-\frac{1}{2}$, and solutions $f r^{n} \mathbf{v}_{0}=s^{n} \mathbf{v}_{0}$ for parameter values $\beta=n+\frac{1}{2}$. We thus obtain, using the aBTs $f$ and $g$, exactly one solution of (6.12) for each half-odd-integer value of $\beta$. We then map back to solutions of (6.10) using the transformation (6.11), to which solutions we can then apply the aBT $k$ if we so wish. The solutions of (6.10) thus derived are generalizations of the matrix solutions of (6.10) obtained from the previously-mentioned upper triangular solutions of (6.12) generated by iterating using $f$ and $g$ from an initial solution (6.15) in the particular case where $\mathbf{v}_{0}$ is obtained from an upper triangular solution of the matrix Airy equation (6.20). That is, they are extensions of a particular case of the matrix analogues of the solutions of the scalar second Painlevé equation expressible in terms of Airy functions obtained above using the initial solution (6.15).

We have thus seen that several extensions to the matrix case of known solutions - rational, expressible in terms of Airy functions - of scalar $P_{I I}$ are possible. Perhaps the most natural are those solution classes found by iteration using aBTs from the initial solutions (6.14) and (6.15). However, it is also possible to seek extensions of these scalar $P_{I I}$ solutions to the matrix case by iterating from (6.23) and (6.24). In this way we find for example solutions of matrix $P_{I I}$ whose elements involve rational and Airy functions. In a later paper we will consider the question of further solution classes, and also extend our results to the case of the matrix $P_{I I}$ hierarchy.

\section{Conclusions}

In this paper we have given an asymptotic reduction from the matrix nonautonomous semidiscrete equation (1.4) to matrix $P_{I}$, as well as corresponding results in the scalar case, that is, a reduction from (1.20) to scalar $P_{I}$. We have also given in Section 4 a more general discussion of our asymptotic analysis, and in Section 5 a component-wise approach. This then extends our earlier papers, where we have explored connections between various integrable systems [20, 21, 22]. In addition, we have given Hamiltonian formulations of the matrix first and second Painlevé equations. Given the results presented in [6] and also in the current paper, we expect in the future to be able to derive further examples of semidiscrete equations using auto-BTs for PDEs and ODEs, and also to be able to obtain asymptotic reductions of 
such new semidiscrete equations to Painlevé equations. Also in the future we will extend our results obtained in Section 6, on classes of solutions of the matrix $P_{I I}$ equation, to the general case of the matrix second Painlevé hierarchy.

\section{Acknowledgments}

We are grateful to the Ministry of Economy and Competitiveness of Spain for funding under grant number MTM2016-80276-P (AEI / FEDER, EU).

\section{References}

[1] PR Gordoa, A Pickering, ZN Zhu. 2016. On matrix Painlevé hierarchies. J. Differential Equations 261, 11281175.

[2] PR Gordoa, A Pickering, ZN Zhu. 2010. Bäcklund transformations for a matrix second Painlevé equation. Phys. Lett. A 374, 3422-3424.

[3] PR Gordoa, A Pickering, ZN Zhu. 2013. Bäcklund transformation of matrix equations and a discrete matrix first Painlevé equation. Phys Lett A 377, 1345-1349.

[4] SP Balandin, VV Sokolov. 1998. On the Painlevé test for non-Abelian equations. Phys Lett A, 246, $267-272$.

[5] PJ Olver, VV Sokolov. 1998. Integrable evolution equations on associative algebras. Commun Math Phys, 193, 245-268.

[6] PR Gordoa, A Pickering. 2018. Auto-Bäcklund transformations for a matrix partial differential equation. Phys Lett A, 382, 1908-1915.

[7] AS Fokas, B Grammaticos, A Ramani. 1993. From continuous to discrete Painlevé equations. J Math Anal Appl, 180, 342-360.

[8] VI Gromak, VV Tsegel'nik, 1994. Functional relations between solutions of equations of P-type. Differents Uravn, 30, 1118-1124; Diff Eqns 30 1037-1043.

[9] D Levi, O Ragnisco, MA Rodríguez. 1993. On non-isospectral flow, Painlevé equations and symmetries of differential and difference equations. Theor Math Phys, 93, 1409-1414.

[10] WX Ma, B Fuchssteiner. 1999. Algebraic structure of discrete zero curvature equations and master symmetries of discrete evolution equations. J Math Phys, 40, 2400-2418.

[11] PR Gordoa, A Pickering, ZN Zhu. 2005. A non-isospectral extension of the Volterra hierarchy to 2+1 dimensions. J Math Phys, 46, 103509.

[12] PR Gordoa, A Pickering, ZN Zhu. 2007. A new 2+1 dimensional non-isospectral Toda lattice hierarchy. J Math Phys, 48, 023515.

[13] WX Ma, Y You. 2004. Rational solutions of the Toda lattice equation in Casoratian form. Chaos, Solitons and Fractals, 22, 395-406.

[14] WX Ma, Y Zhou. 2018. Lump solutions to nonlinear partial differential equations via Hirota bilinear forms. $J$ Differential Equations, 264, 2633-2659.

[15] WX Ma, X Yong, HQ Zhang. 2018. Diversity of interaction solutions to the (2+1)-dimensional Ito equation. Computers and Mathematics with Applications, 75, 289-295.

[16] J Villarroel. 1998. On the solution to the inverse problem for the Toda chain. SIAM J Appl Math, 59, $261-285$.

[17] K. Okamoto. 1999. The Hamiltonians associated to the Painlevé equations, in "The Painlevé Property. One Century Later," ed. R. Conte (CRM Series in Mathematical Physics, Springer-Verlag, New York), $735-787$.

[18] $\mathrm{RH}$ Bartels, GW Stewart. 1972. Algorithm 432: Solution of the matrix equation $A X+X B=C$. Comm. $A C M$, 15, 820-826.

[19] G Kitagawa. 1977. An algorithm for solving the matrix equation $X=F X F^{T}+S$. Int J Control, 25, 745-753.

[20] A Pickering, PR Gordoa, JAD Wattis. 2014. Behaviour of the extended Volterra lattice., Commun Nonlinear Sci Numer Simulat, 19, 589-600.

[21] JAD Wattis, PR Gordoa, A Pickering. 2015. Behaviour of the extended Toda lattice. Commun Nonlinear Sci Numer Simul, 28, 138-150,

[22] JAD Wattis, PR Gordoa, A Pickering. 2018. Behaviour of the extended modified Volterra lattice - Reductions to generalised mKdV and NLS equations. Commun Nonlinear Sci Numer Simulat, 65, 98-110. 